\title{
FULLY-DISCRETE FINITE ELEMENT APPROXIMATIONS FOR A FOURTH-ORDER LINEAR STOCHASTIC PARABOLIC EQUATION WITH ADDITIVE SPACE-TIME WHITE NOISE*
}

\author{
Georgios T. Kossioris $^{1}$ And Georgios E. Zouraris ${ }^{1}$
}

\begin{abstract}
We consider an initial and Dirichlet boundary value problem for a fourth-order linear stochastic parabolic equation, in one space dimension, forced by an additive space-time white noise. Discretizing the space-time white noise a modelling error is introduced and a regularized fourth-order linear stochastic parabolic problem is obtained. Fully-discrete approximations to the solution of the regularized problem are constructed by using, for discretization in space, a Galerkin finite element method based on $\mathrm{C}^{0}$ or $\mathrm{C}^{1}$ piecewise polynomials, and, for time-stepping, the Backward Euler method. We derive strong a priori estimates for the modelling error and for the approximation error to the solution of the regularized problem.
\end{abstract}

Mathematics Subject Classification. 65M60,65M15, 65C20.

Received April 19, 2007. Revised May 20, 2009.

Published online January 27, 2010.

\section{INTRODUCTION}

\subsection{The main problem}

Let $T>0, D=(0,1)$ and $(\Omega, \mathcal{F}, P)$ be a complete probability space. Then we consider an initial and Dirichlet boundary value problem for a fourth-order linear stochastic parabolic equation which is formulated, typically, as follows: find a stochastic function $u:[0, T] \times \bar{D} \rightarrow \mathbb{R}$ such that

$$
\begin{gathered}
\partial_{t} u+\partial_{x}^{4} u=\partial_{t x} W(t, x) \quad \forall(t, x) \in(0, T] \times D, \\
\left.\partial_{x}^{2 m} u(t, \cdot)\right|_{\partial D}=0 \quad \forall t \in(0, T], \quad m=0,1, \\
u(0, x)=0 \quad \forall x \in D,
\end{gathered}
$$

\footnotetext{
Keywords and phrases. Finite element method, space-time white noise, Backward Euler time-stepping, fully-discrete approximations, a priori error estimates.

* Work supported by The Research Committee and The Research Account of The University of Crete under the Research Project no.2299: "Analysis of numerical methods for the approximation of the solution of stochastic and non-stochastic partial differential equations of parabolic type" (2006-2007).

${ }^{1}$ Department of Mathematics, University of Crete, 71409 Heraklion, Crete, Greece and Institute of Applied and Computational Mathematics, FO.R.T.H., 71110 Heraklion, Crete, Greece. kosioris@math.uoc.gr; zouraris@math.uoc.gr
} 
a.s. in $\Omega$, where $\partial_{t x} W$ denotes a space-time white noise on $[0, T] \times D$ (see, e.g., [17,28]). The mild solution of the problem above $(c f .[7,10])$ (known also as 'stochastic convolution') is given by the formula

$$
u(t, x)=\int_{0}^{t} \int_{D} G(t-s ; x, y) \mathrm{d} W(s, y) .
$$

Here, $G(t ; x, y)$ is the space-time Green kernel of the corresponding deterministic parabolic problem: find a deterministic function $w:[0, T] \times \bar{D} \rightarrow \mathbb{R}$ such that

$$
\begin{gathered}
\partial_{t} w+\partial_{x}^{4} w=0 \quad \forall(t, x) \in(0, T] \times D, \\
\left.\partial_{x}^{2 m} w(t, \cdot)\right|_{\partial D}=0 \quad \forall t \in(0, T], \quad m=0,1, \\
w(0, x)=w_{0}(x) \quad \forall x \in D,
\end{gathered}
$$

where $w_{0}$ is a deterministic initial condition. In particular, we have

$$
w(t, x)=\int_{D} G(t ; x, y) w_{0}(y) \mathrm{d} y \quad \forall(t, x) \in(0, T] \times \bar{D}
$$

and

$$
G(t ; x, y)=\sum_{k=1}^{\infty} \mathrm{e}^{-\lambda_{k}^{4} t} \varepsilon_{k}(x) \varepsilon_{k}(y) \quad \forall(t, x, y) \in(0, T] \times \bar{D} \times \bar{D}
$$

where $\lambda_{k}:=k \pi$ for $k \in \mathbb{N}$, and $\varepsilon_{k}(z):=\sqrt{2} \sin \left(\lambda_{k} z\right) \quad \forall z \in \bar{D}, \quad \forall k \in \mathbb{N}$.

\subsection{The regularized problem}

Being inspired by the approach for a second order stochastic parabolic equation with additive space-time white noise proposed in [1], we introduce in this section an approximate regularized initial and boundary value problem which we will use to derive computable approximations to the solution $u$ of (1.1).

Let $N_{\Delta} \in \mathbb{N}$ and $\left(t_{n}\right)_{n=0}^{N_{\Delta}}$ be the nodes of a partition of $[0, T]$. Then, define $T_{n}:=\left(t_{n-1}, t_{n}\right)$ and $\Delta t_{n}:=$ $t_{n}-t_{n-1}$ for $n=1, \ldots, N_{\Delta}$. Also, let $J_{\Delta} \in \mathbb{N}$ and $\left(x_{j}\right)_{j=0}^{J_{\Delta}}$ be the nodes of a partition of $\bar{D}$. Then, define $x_{j-\frac{1}{2}}:=\frac{1}{2}\left(x_{j-1}+x_{j}\right), D_{j}:=\left(x_{j-1}, x_{j}\right)$ and $\Delta x_{j}:=x_{j}-x_{j-1}$ for $j=1, \ldots, J_{\Delta}$. Also, set $\Delta t:=\max _{1 \leq n \leq N_{\Delta}} \Delta t_{n}$ and $\Delta x:=\max _{1 \leq j \leq J_{\Delta}} \Delta x_{j}$. Next, for $j=1, \ldots, J_{\Delta}$, specify an $\mathrm{L}^{2}\left(D_{j}\right)$-orthogonal basis $\left\{\widehat{\pi}_{1, j}, \widehat{\pi}_{2, j}\right\}$ of $\mathbb{P}^{1}\left(D_{j}\right)$ with $\widehat{\pi}_{1, j}:=\frac{1}{\sqrt{\Delta x_{j}}}$ and $\widehat{\pi}_{2, j}:=\frac{2 \sqrt{3}}{\left(\Delta x_{j}\right)^{\frac{3}{2}}}\left(x-x_{j-\frac{1}{2}}\right)$. Finally, consider the fourth-order linear stochastic parabolic problem: find a stochastic function $\widehat{u}:[0, T] \times \bar{D} \rightarrow \mathbb{R}$ such that

$$
\begin{gathered}
\partial_{t} \widehat{u}+\partial_{x}^{4} \widehat{u}=\widehat{W} \quad \text { in }(0, T] \times D, \\
\left.\partial_{x}^{2 m} \widehat{u}(t, \cdot)\right|_{\partial D}=0 \quad \forall t \in(0, T], \quad m=0,1, \\
\widehat{u}(0, x)=0 \quad \forall x \in D,
\end{gathered}
$$

a.e. in $\Omega$, where

$$
\begin{gathered}
\widehat{W}(t, x):=\sum_{n=1}^{N_{\Delta}} \frac{1}{\Delta t_{n}} \sum_{j=1}^{J_{\Delta}} \mathcal{X}_{S_{n, j}}(t, x)\left[R_{n, 1, j} \widehat{\pi}_{1, j}(x)+R_{n, 2, j} \widehat{\pi}_{2, j}(x)\right] \quad \forall(t, x) \in[0, T] \times \bar{D}, \\
R_{n, i, j}:=\int_{S_{n, j}} \widehat{\pi}_{i, j}(x) \mathrm{d} W(t, x),
\end{gathered}
$$


and $S_{n, j}:=T_{n} \times D_{j}$ for $j=1, \ldots, J_{\Delta}$ and $n=1, \ldots, N_{\Delta}$. It is well known that the solution of the problem (1.6), according to the standard theory for parabolic problems (see, e.g., [21]), has the integral representation

$$
\widehat{u}(t, x)=\int_{0}^{t} \int_{D} G(t-s ; x, y) \widehat{W}(s, y) \mathrm{d} s \mathrm{~d} y \quad \forall(t, x) \in[0, T] \times \bar{D} .
$$

We next approximate the mild solution $u$ of (1.1) by constructing numerical approximations to the solution $\widehat{u}$ of (1.6), which is a usual parabolic problem with stochastic load. Hence, the approach above results in two kind of errors that we have to estimate: (i) the, so called, modelling error which is the difference $u-\widehat{u}$, and (ii) the numerical approximation error for $\widehat{u}$.

Remark 1.1. Let $\mathcal{I}=\left\{(n, i, j): n=1, \ldots, N_{\Delta}, i=1,2, j=1, \ldots, J_{\Delta}\right\}$. Using the properties of the stochastic integral (see, e.g., [28]), we conclude that $R_{\beta} \sim \mathcal{N}\left(0, \Delta t_{\beta_{1}}\right.$ ) for all $\beta \in \mathcal{I}$; also, we observe that $\mathbb{E}\left[R_{\beta} R_{\beta^{\prime}}\right]=0$ for $\beta, \beta^{\prime} \in \mathcal{I}$ with $\beta \neq \beta^{\prime}$. Thus, the random variables $\left(R_{\beta}\right)_{\beta \in \mathcal{I}}$ are independent since they are Gaussian and uncorrelated.

\subsection{Main results of the paper}

The motivation to consider the problem (1.1) is driven by the fact that its solution is part of the mild solution of the stochastic Cahn-Hilliard equation ( $c f$. [7,10]). The latter equation was introduced by Cook [9] for the investigation of phase separation in spinodal decomposition (see, e.g., [12,18]). In the paper at hand, our plan is to propose fully-discrete Backward Euler finite element methods to approximate the solution of (1.6), and analyze their convergence to the mild solution (1.2) of (1.1). In spite of the recent contributions in the convergence analysis of the finite element method for second order stochastic parabolic problems with additive space-time white noise (see, e.g., $[1,3,14,19,25,27,29]$ ), the order of convergence of the finite element method for the fourth order problem (1.1) is not conclusive. Also, we refer to [6] for the convergence analysis of a finite difference method, to $[15,16,23]$ for the convergence analysis of time-discretization methods for a wide family of evolution problems that includes (1.1), while the finite element method is not among the space-discretization techniques considered in $[15,16]$.

The first result of the paper is the derivation of a convergent bound for the modelling error. Indeed, using the integral representation of $u$ and $\widehat{u}$, we obtain (see Thm. 3.1) the comparison estimate

$$
\max _{t \in[0, T]}\left\{\int_{\Omega}\left(\int_{D}|u(t, x)-\widehat{u}(t, x)|^{2} \mathrm{~d} x\right) \mathrm{d} P\right\}^{\frac{1}{2}} \leq C\left(\Delta t^{\frac{3}{8}}+\epsilon^{-\frac{1}{2}} \Delta x^{\frac{3}{2}-\epsilon}\right), \quad \forall \epsilon \in\left(0, \frac{3}{2}\right],
$$

which measures the effect of discretizing the space-time white noise by the discrete space-time white noise kernel $\widehat{W}$. The error bound (1.9) validates the decision to approximate $u$ via the construction of approximations to $\widehat{u}$, because it concludes that $\widehat{u}$ tends to $u$ when $\Delta t$ and $\Delta x$ tend to zero.

Let $M \in \mathbb{N}$ and $\left(\tau_{m}\right)_{m=0}^{M}$ be the nodes of a partition of $[0, T]$, i.e. $\tau_{0}=0, \tau_{M}=T$ and $\tau_{m-1}<\tau_{m}$ for $m=1, \ldots, M$. Then, we define $\Delta_{m}:=\left(\tau_{m-1}, \tau_{m}\right)$ and $k_{m}:=\tau_{m}-\tau_{m-1}$ for $m=1, \ldots, M$, and set $k_{\max }:=\max _{1 \leq m \leq M} k_{m}$. Also, for $r \in\{1,2,3\}$ and $\kappa(r) \in\{1, \ldots, r\}$, let $S_{h}^{r, \kappa(r)} \subset \mathrm{H}_{0}^{1}(D) \cap \mathrm{H}^{\kappa(r)}(D)$ be a finite element space consisting of functions which are piecewise polynomials of degree at most $r$ over a partition of $D$ in intervals with maximum mesh-length $h$. Then, we define a discrete Laplace operator $\Delta_{h}: S_{h}^{r, \kappa(r)} \rightarrow S_{h}^{r, \kappa(r)}$ by

$$
\left(\Delta_{h} \varphi, \chi\right)_{0, D}=\left(\varphi^{\prime}, \chi^{\prime}\right)_{0, D} \quad \forall \varphi, \chi \in S_{h}^{r, \kappa(r)},
$$

and, when $k(r) \geq 2$, a discrete biharmonic operator $B_{h}: S_{h}^{r, \kappa(r)} \rightarrow S_{h}^{r, \kappa(r)}$ by

$$
\left(B_{h} \varphi, \chi\right)_{0, D}=\left(\varphi^{\prime \prime}, \chi^{\prime \prime}\right)_{0, D} \quad \forall \varphi, \chi \in S_{h}^{r, \kappa(r)} .
$$


Also, the usual $\mathrm{L}^{2}(D)$-projection operator $P_{h}: \mathrm{L}^{2}(D) \rightarrow S_{h}^{r, \kappa(r)}$ is specified by

$$
\left(P_{h} f, \chi\right)_{0, D}=(f, \chi)_{0, D} \quad \forall \chi \in S_{h}^{r, \kappa(r)}, \quad \forall f \in \mathrm{L}^{2}(D) .
$$

Computable fully-discrete approximations of $\widehat{u}$ are constructed by using the Backward Euler finite element method described below:

Step FD1. Set

$$
\widehat{U}_{h}^{0}:=0
$$

Step FD2. For $m=1, \ldots, M$, find $\widehat{U}_{h}^{m} \in S_{h}^{r, \kappa(r)}$ such that

$$
\widehat{U}_{h}^{m}-\widehat{U}_{h}^{m-1}+k_{m} Q_{h} \widehat{U}_{h}^{m}=\int_{\Delta_{m}} P_{h} \widehat{W} \mathrm{~d} s
$$

where $Q_{h}=\Delta_{h}^{2}$ or $Q_{h}=B_{h}$.

For the numerical method above, the first convergence result we obtain (see Thm. 6.3) is the discrete in time $\mathrm{L}_{t}^{2}\left(\mathrm{~L}_{P}^{2}\left(\mathrm{~L}_{x}^{2}\right)\right)$ error estimate:

$$
\left\{\sum_{m=1}^{M} k_{m} \int_{\Omega}\left(\int_{D}\left|\widehat{U}_{h}^{m}(x)-\widehat{u}\left(\tau_{m}, x\right)\right|^{2} \mathrm{~d} x\right) \mathrm{d} P\right\}^{\frac{1}{2}} \leq C\left[\left(k_{\max }\right)^{\frac{3}{8}}+\epsilon^{-\frac{1}{2}} h^{\nu-\epsilon}\right], \quad \forall \epsilon \in(0, \nu],
$$

where: $\nu$ depends on $r, \kappa(r)$, the biharmonic operator $Q_{h}$ chosen and the properties of the finite element spaces (see Thm. 5.3). Adopting a technique based on energy estimates for non-homogeneous deterministic parabolic problems, we derive (see Thm. 6.5) the alternative discrete in time $\mathrm{L}_{t}^{2}\left(\mathrm{~L}_{P}^{2}\left(\mathrm{~L}_{x}^{2}\right)\right.$ )-error estimate given below:

$$
\left\{\sum_{m=1}^{M} k_{m} \int_{\Omega}\left(\int_{D}\left|\widehat{U}_{h}^{m}(x)-\widehat{u}\left(\tau_{m}, x\right)\right|^{2} \mathrm{~d} x\right) \mathrm{d} P\right\}^{\frac{1}{2}} \leq C\left[\left(k_{\max }\right)^{\frac{3}{8}}+h^{r}+h^{r} \Delta t^{-\frac{3}{8}}\right] .
$$

Assuming that the nodes $\left(\tau_{m}\right)_{m=0}^{M}$ are equidistributed with $k_{m}=\Delta \tau$ for $m=1, \ldots, M$, and using Duhamel's principle we arrive at the following discrete in time $\mathrm{L}_{t}^{\infty}\left(\mathrm{L}_{P}^{2}\left(\mathrm{~L}_{x}^{2}\right)\right)$ error estimate (see Thm. 6.9):

$$
\max _{0 \leq m \leq M}\left\{\int_{\Omega}\left(\int_{D}\left|\widehat{U}_{h}^{m}(x)-\widehat{u}\left(\tau_{m}, x\right)\right|^{2} \mathrm{~d} x\right) \mathrm{d} P\right\}^{\frac{1}{2}} \leq C\left(\epsilon_{1}^{-\frac{1}{2}} \Delta \tau^{\frac{3}{8}-\epsilon_{1}}+\epsilon_{2}^{-\frac{1}{2}} h^{\nu-\epsilon_{2}}\right),
$$

for $\epsilon_{1} \in\left(0, \frac{3}{8}\right]$ and $\epsilon_{2} \in(0, \nu]$. Finally, applying an energy estimate technique we get the following alternative discrete in time $\mathrm{L}_{t}^{\infty}\left(\mathrm{L}_{P}^{2}\left(\mathrm{~L}_{x}^{2}\right)\right)$ error estimate (see Thm. 6.10):

$$
\max _{0 \leq m \leq M}\left\{\int_{\Omega}\left(\int_{D}\left|\widehat{U}_{h}^{m}(x)-\widehat{u}\left(\tau_{m}, x\right)\right|^{2} \mathrm{~d} x\right) \mathrm{d} P\right\}^{\frac{1}{2}} \leq C\left(\epsilon^{-\frac{1}{2}} \Delta \tau^{\frac{3}{8}-\epsilon}+h^{r} \Delta \tau^{-1}\right),
$$

for $\epsilon \in\left(0, \frac{3}{8}\right]$.

We close this section with some remarks related to the error estimates above:

- To obtain the error estimate (1.14), we introduce a space-discrete approximation $\widehat{u}_{h}$ of $\widehat{u}$ and analyze its convergence in the $\mathrm{L}_{t}^{\infty}\left(\mathrm{L}_{P}^{2}\left(\mathrm{~L}_{x}^{2}\right)\right)$ norm (see Thm. 5.3).

- Although the estimates (1.16) and (1.17) are of stronger norm, the estimates (1.14) and (1.15) have their own value since the order of convergence is slightly better and they allow nonuniform partition of the time interval, thus, they are naturally suited for designing adaptive methods. Following the 
adopted techniques, such estimates can be also derived for the second order equations, for which, to our knowledge, there are no analogue results in the existing literature (cf. $[3,15,16,25,27,29])$.

- A first impression is that, estimates (1.14) and (1.16), are qualitatively better than the estimates (1.15) and (1.17), respectively, because no negative powers of $\Delta t$ or $\Delta \tau$ appear. However, choosing $h=O(\Delta t)$, the estimate (1.15) becomes of order $r-\frac{3}{8}$ with respect to $h$, which is greater than $\nu$. Also, choosing $h=O(\Delta \tau)$ the estimate (1.17) becomes of order $r-1$ with respect to $h$, which is greater than $\nu$ for $r=3$ or 2. A fair comparison could be done, by estimating the total work needed to compute an approximation of a statistical quantity of $u$ with error below of a given tolerance (see, e.g., $[2,22]$ ), which is beyond the scope of the present work.

- Error estimates, in the discrete in time $\mathrm{L}_{t}^{\infty}\left(\mathrm{L}_{P}^{2}\left(\mathrm{~L}_{x}^{2}\right)\right)$ norm, for Backward Euler fully-discrete finite element approximations, but for the stochastic heat equation with additive space-time white noise, have been proved recently by Yan [25,27] and Bin [3]. Here, to get the estimates (1.16) and (1.17) we adopt a different point of view. First we consider the Backward-Euler time-discrete approximations of $\widehat{u}$ and analyze their convergence to $\widehat{u}$ in the discrete in time $\mathrm{L}_{t}^{\infty}\left(\mathrm{L}_{P}^{2}\left(\mathrm{~L}_{x}^{2}\right)\right.$ ) norm (see Thm. 4.2). Then, we compare the Backward-Euler fully-discrete approximations of $\widehat{u}$ with the Backward-Euler time-discrete approximations of $\widehat{u}$ (see Prop. 6.8 and Thm. 6.10). Thus, we are able to estimate separately the error contribution in time due to the Backward Euler time-stepping and the error contribution in space due to the finite element method.

\subsection{Overview}

We close the section by an overview of the paper. Section 2 introduces notation, includes some known results often used in the paper, recalls properties of continuous parabolic and elliptic solution operators, describes our finite element spaces and exposes some error estimates for finite element approximations of the solution of an one-dimensional biharmonic elliptic problem. Section 3 is dedicated to the estimation of the modelling error. Section 4 defines the Backward Euler time-discrete approximations of $\widehat{u}$ and analyzes its convergence. Section 5 defines a finite element space-discrete approximation of $\widehat{u}$ and estimates its approximation error. Section 6 contains the error analysis for the Backward Euler fully-discrete approximations of $\widehat{u}$.

\section{Notation AND PRELIMINARIES}

\subsection{Function spaces and related operators}

Let $I \subset \mathbb{R}$ be a bounded interval. We denote by $\mathrm{L}^{2}(I)$ the space of the Lebesgue measurable functions which are square integrable on $I$ with respect to Lebesgue's measure $\mathrm{d} x$, provided with the standard norm $\|g\|_{0, I}:=\left\{\int_{I}|g(x)|^{2} \mathrm{~d} x\right\}^{\frac{1}{2}}$ for $g \in \mathrm{L}^{2}(I)$. The standard inner product in $\mathrm{L}^{2}(I)$ that produces the norm $\|\cdot\|_{0, I}$ is written as $(\cdot, \cdot)_{0, I}$, i.e., $\left(g_{1}, g_{2}\right)_{0, I}:=\int_{I} g_{1}(x) g_{2}(x) \mathrm{d} x$ for $g_{1}, g_{2} \in \mathrm{L}^{2}(I)$. For $s \in \mathbb{N}_{0}, \mathrm{H}^{s}(I)$ will be the Sobolev space of functions having generalized derivatives up to order $s$ in the space $\mathrm{L}^{2}(I)$, and by $\|\cdot\|_{s, I}$ its usual norm, i.e. $\|g\|_{s, I}:=\left\{\sum_{\ell=0}^{s}\left\|\partial^{\ell} g\right\|_{0, I}^{2}\right\}^{\frac{1}{2}}$ for $g \in \mathrm{H}^{s}(I)$. Also, by $\mathrm{H}_{0}^{1}(I)$ we denote the subspace of $\mathrm{H}^{1}(I)$ consisting of functions which vanish at the endpoints of $I$ in the sense of trace. We note that in $\mathrm{H}_{0}^{1}(I)$ the, well-known, Poincaré-Friedrich inequality holds, i.e.,

$$
\|g\|_{0, I} \leq C_{P F}\left\|g^{\prime}\right\|_{0, I} \quad \forall g \in \mathrm{H}_{0}^{1}(I) .
$$

To simplify the notation we set $\mathbb{H}^{s}(D):=\mathrm{H}^{s}(D) \cap \mathrm{H}_{0}^{1}(D)$ for $s \in \mathbb{N}$.

The sequence of pairs $\left\{\left(\lambda_{k}^{2}, \varepsilon_{k}\right)\right\}_{k=1}^{\infty}$ is a solution to the eigenvalue/eigenfunction problem: find nonzero $\varphi \in \mathbb{H}^{2}(D)$ and $\sigma \in \mathbb{R}$ such that $-\varphi^{\prime \prime}=\sigma \varphi$ in $D$. Since $\left(\varepsilon_{k}\right)_{k=1}^{\infty}$ is a complete $(\cdot, \cdot)_{0, D}$-orthonormal system 
in $\mathrm{L}^{2}(D)$, for $s \in \mathbb{R}$, a subspace $\dot{\mathbf{H}}^{s}(D)$ of $\mathrm{L}^{2}(D)$ (see [24]) is defined by

$$
\dot{\mathbf{H}}^{s}(D):=\left\{v \in \mathrm{L}^{2}(D): \sum_{k=1}^{\infty} \lambda_{k}^{2 s}\left(v, \varepsilon_{k}\right)_{0, D}^{2}<\infty\right\}
$$

and provided with the norm $\|v\|_{\dot{\mathbf{H}}^{s}}:=\left(\sum_{k=1}^{\infty} \lambda_{k}^{2 s}\left(v, \varepsilon_{k}\right)_{0, D}^{2}\right)^{\frac{1}{2}} \quad \forall v \in \dot{\mathbf{H}}^{s}(D)$. Let $m \in \mathbb{N}_{0}$. It is well-known (see [24]) that

$$
\dot{\mathbf{H}}^{m}(D)=\left\{v \in H^{m}(D):\left.\quad \partial_{x}^{2 i} v\right|_{\partial D}=0 \quad \text { if } 0 \leq i<\frac{m}{2}\right\}
$$

and there exist constants $C_{m, A}$ and $C_{m, B}$ such that

$$
C_{m, A}\|v\|_{m, D} \leq\|v\|_{\dot{\mathbf{H}}^{m}} \leq C_{m, B}\|v\|_{m, D} \quad \forall v \in \dot{\mathbf{H}}^{m}(D) .
$$

Also, we define on $\mathrm{L}^{2}(D)$ the negative norm $\|\cdot\|_{-m, D}$ by

$$
\|v\|_{-m, D}:=\sup \left\{\frac{(v, \varphi)_{0, D}}{\|\varphi\|_{m, D}}: \quad \varphi \in \dot{\mathbf{H}}^{m}(D) \text { and } \varphi \neq 0\right\} \quad \forall v \in \mathrm{L}^{2}(D),
$$

for which, using (2.3), it is easy to conclude that there exists a constant $C_{-m}>0$ such that

$$
\|v\|_{-m, D} \leq C_{-m}\|v\|_{\dot{\mathrm{H}}^{-m}} \quad \forall v \in \mathrm{L}^{2}(D) .
$$

Let $\mathbb{L}_{2}=\left(\mathrm{L}^{2}(D),(\cdot, \cdot)_{0, D}\right)$ and $\mathcal{L}\left(\mathbb{L}_{2}\right)$ be the space of linear, bounded operators from $\mathbb{L}_{2}$ to $\mathbb{L}_{2}$. We say that, an operator $\Phi \in \mathcal{L}\left(\mathbb{L}_{2}\right)$ is Hilbert-Schmidt, when $\|\Phi\|_{\mathrm{HS}}:=\left\{\sum_{k=1}^{\infty}\left\|\Phi\left(\varepsilon_{k}\right)\right\|_{0, D}^{2}\right\}^{\frac{1}{2}}<+\infty$, where $\|\Phi\|_{\mathrm{HS}}$ is the so called Hilbert-Schmidt norm of $\Phi$. We note that the quantity $\|\Phi\|_{\text {HS }}$ does not change when we replace $\left(\varepsilon_{k}\right)_{k=1}^{\infty}$ by another complete orthonormal system of $\mathbb{L}_{2}$. It is well known (see, e.g., [11]) that an operator $\Phi \in \mathcal{L}\left(\mathbb{L}_{2}\right)$ is Hilbert-Schmidt iff there exists a measurable function $g: D \times D \rightarrow \mathbb{R}$ such that $\Phi[v](\cdot)=\int_{D} g(\cdot, y) v(y) \mathrm{d} y$ for $v \in \mathrm{L}^{2}(D)$, and then, it holds that

$$
\|\Phi\|_{\mathrm{HS}}=\left(\int_{D} \int_{D} g^{2}(x, y) \mathrm{d} x \mathrm{~d} y\right)^{\frac{1}{2}}
$$

Let $\mathcal{L}_{\mathrm{HS}}\left(\mathbb{L}_{2}\right)$ be the set of Hilbert Schmidt operators of $\mathcal{L}\left(\mathbb{L}^{2}\right)$ and $\widehat{\Phi}:[0, T] \rightarrow \mathcal{L}_{\mathrm{HS}}\left(\mathbb{L}_{2}\right)$. Also, for a random variable $X$, let $\mathbb{E}[X]$ be its expected value, i.e., $\mathbb{E}[X]:=\int_{\Omega} X \mathrm{~d} P$. Then, the Itô isometry property for stochastic integrals, which we will use often in the paper, reads

$$
\mathbb{E}\left[\left\|\int_{0}^{T} \widehat{\Phi} \mathrm{d} W\right\|_{0, D}^{2}\right]=\int_{0}^{T}\|\widehat{\Phi}(t)\|_{\mathrm{HS}}^{2} \mathrm{~d} t .
$$

We close this section by observing that: if $c_{\star}>0$, then

$$
\sum_{k=1}^{\infty} \lambda_{k}^{-\left(1+c_{\star} \epsilon\right)} \leq\left(\frac{1+2 c_{\star}}{c_{\star} \pi}\right) \frac{1}{\epsilon} \quad \forall \epsilon \in(0,2] .
$$

\subsection{Discrete space-time white noise kernel $\widehat{W}$}

In the rest of the paper, we will, often, use the projection operator $\widehat{\Pi}: \mathrm{L}^{2}((0, T) \times D) \rightarrow \mathrm{L}^{2}((0, T) \times D)$ defined by

$$
\left.\widehat{\Pi} g\right|_{S_{n, j}}:=\frac{1}{\Delta t_{n}} \sum_{i=1}^{2}\left(\int_{S_{n, j}} g(t, y) \widehat{\pi}_{i, j}(y) \mathrm{d} t \mathrm{~d} y\right) \widehat{\pi}_{i, j}, \quad n=1, \ldots, N_{\Delta}, \quad j=1, \ldots, J_{\Delta},
$$


for $g \in \mathrm{L}^{2}((0, T) \times D)$, for which obviously holds that

$$
\left(\int_{0}^{T} \int_{D}(\widehat{\Pi} g)^{2} \mathrm{~d} x \mathrm{~d} t\right)^{\frac{1}{2}} \leq\left(\int_{0}^{T} \int_{D} g^{2} \mathrm{~d} x \mathrm{~d} t\right)^{\frac{1}{2}} \quad \forall g \in \mathrm{L}^{2}((0, T) \times D) .
$$

Next lemma, relates the stochastic integral of the projection $\widehat{\Pi}$ of a deterministic function to its space-time $\mathrm{L}^{2}$-inner product with the discrete space-time white noise kernel $\widehat{W}$ defined in Section 1.2.

Lemma 2.1. For $g \in \mathrm{L}^{2}((0, T) \times D)$, it holds that

$$
\int_{0}^{T} \int_{D} \widehat{\Pi} g(s, y) \mathrm{d} W(s, y)=\int_{0}^{T} \int_{D} \widehat{W}(t, x) g(t, x) \mathrm{d} t \mathrm{~d} x .
$$

Proof. To obtain (2.10) we work, using (2.8) and the properties of the stochastic integral, as follows:

$$
\begin{aligned}
\int_{0}^{T} \int_{D} \widehat{\Pi} g(s, y) \mathrm{d} W(s, y) & =\sum_{n=1}^{N_{\Delta}} \sum_{j=1}^{J_{\Delta}} \sum_{i=1}^{2} \frac{1}{\Delta t_{n}}\left(\int_{S_{n, j}} g(t, x) \widehat{\pi}_{i, j}(x) \mathrm{d} t \mathrm{~d} x\right) R_{n, i, j} \\
& =\sum_{n=1}^{N_{\Delta}} \sum_{j=1}^{J_{\Delta}} \frac{1}{\Delta t_{n}} \int_{S_{n, j}} g(t, x)\left(\sum_{i=1}^{2} \widehat{\pi}_{i, j}(x) R_{n, i, j}\right) \mathrm{d} t \mathrm{~d} x \\
& =\sum_{n=1}^{N_{\Delta}} \sum_{j=1}^{J_{\Delta}} \frac{1}{\Delta t_{n}} \int_{0}^{T} \int_{D} \mathcal{X}_{S_{n, j}}(t, x) g(t, x)\left(\sum_{i=1}^{2} \widehat{\pi}_{i, j}(x) R_{n, i, j}\right) \mathrm{d} t \mathrm{~d} x \\
& =\int_{0}^{T} \int_{D} g(t, x) \widehat{W}(t, x) \mathrm{d} t \mathrm{~d} x
\end{aligned}
$$

\subsection{Linear elliptic and parabolic operators}

First, we consider the following Dirichlet two-point boundary value problem: for given $f \in \mathrm{L}^{2}(D)$ find $v_{E} \in \dot{\mathbf{H}}^{2}(D)$ such that

$$
v_{E}^{\prime \prime}=f \quad \text { in } D
$$

We will denote by $T_{E}: \mathrm{L}^{2}(D) \rightarrow \dot{\mathbf{H}}^{2}(D)$ the solution operator of (2.11), i.e. $T_{E} f:=v_{E}$, which has the property

$$
\left\|T_{E} f\right\|_{m, D} \leq C\|f\|_{m-2, D}, \quad \forall f \in \mathrm{H}^{\max \{0, m-2\}}(D), \quad \forall m \in \mathbb{N}_{0} .
$$

Consider, also, the following Dirichlet biharmonic two-point boundary value problem: given $f \in \mathrm{L}^{2}(D)$ find $v_{B} \in \dot{\mathbf{H}}^{4}(D)$ such that

$$
v_{B}^{\prime \prime \prime \prime}=f \quad \text { in } D
$$

Letting $T_{B}: \mathrm{L}^{2}(D) \rightarrow \dot{\mathbf{H}}^{4}(D)$ be the solution operator of $(2.13)$, i.e. $T_{B} f:=v_{B}$, it is easy to verify that

$$
\left\|T_{B} f\right\|_{m, D} \leq C\|f\|_{m-4, D}, \quad \forall f \in \mathrm{H}^{\max \{0, m-4\}}(D), \quad \forall m \in \mathbb{N}_{0} .
$$

Due to the type of boundary conditions of (2.13), we conclude that

$$
T_{B} f=T_{E}^{2} f, \quad \forall f \in \mathrm{L}^{2}(D),
$$

which, easily, yields

$$
\left(T_{B} v_{1}, v_{2}\right)_{0, D}=\left(T_{E} v_{1}, T_{E} v_{2}\right)_{0, D} \quad \forall v_{1}, v_{2} \in \mathrm{L}^{2}(D)
$$

Let $\left(\mathcal{S}(t) w_{0}\right)_{t \in[0, T]}$ be the standard semigroup notation for the solution $w$ of (1.3). For later use, we display some a priori bounds for norms of $w$ with respect to the initial data $w_{0}$, obtained by proceeding as in the proof 
of analogous results for linear second order deterministic parabolic problems (see Chap. 3 in [20,24]): for $\ell \in \mathbb{N}_{0}$, $\beta, p \in \mathbb{R}_{0}^{+}$and $q \in[0, p+4 \ell]$ there exists a constant $C_{p, q, \ell}>0$ such that

$$
\left\|\partial_{t}^{\ell} \mathcal{S}(t) w_{0}\right\|_{\dot{H}^{p}} \leq C_{p, q, \ell} t^{-\frac{p-q}{4}-\ell}\left\|w_{0}\right\|_{\dot{\mathbf{H}}^{q}} \quad \forall t>0, \quad \forall w_{0} \in \dot{\mathbf{H}}^{q}(D),
$$

and a constant $C_{\beta}>0$ such that

$$
\int_{t_{a}}^{t_{b}}\left(\tau-t_{a}\right)^{\beta}\left\|\partial_{t}^{\ell} \mathcal{S}(\tau) w_{0}\right\|_{\dot{\mathbf{H}}^{p}}^{2} \mathrm{~d} \tau \leq C_{\beta}\left\|w_{0}\right\|_{\dot{\mathbf{H}}^{p+4 \ell-2 \beta-2}}^{2} \quad \forall t_{b}>t_{a} \geq 0, \quad \forall w_{0} \in \dot{\mathbf{H}}^{p+4 \ell-2 \beta-2}(D) .
$$

\subsection{Finite element spaces and approximations}

Let $r \in\{1,2,3\}$ and $\kappa(r) \in\{1, \ldots, r\}$. Then, we consider a finite element space $S_{h}^{r, \kappa(r)} \subset \mathbb{H}^{\kappa(r)}(D)$ consisting of functions which are piecewise polynomials of degree at most $r$ over a partition of $D$ in intervals with maximum mesh-length $h$. It is well-known ( $c f .$, e.g., $[4,5,8])$ that the following approximation properties hold:

$$
\inf _{\chi \in S_{h}^{r, \kappa(r)}}\left(\|v-\chi\|_{0, D}+h\|v-\chi\|_{1, D}\right) \leq C h^{\ell}\|v\|_{\ell, D} \quad \forall v \in \mathbb{H}^{\ell}(D), \quad \ell=2, \ldots, r+1,
$$

and

$$
\inf _{\chi \in S_{h}^{r, \kappa(r)}}\|v-\chi\|_{2, D} \leq C h^{s-1}\|v\|_{s+1, D} \quad \forall v \in \mathbb{H}^{s+1}(D), \quad s=2, \ldots, r, \quad r=2,3, \quad \kappa(r) \geq 2 .
$$

If the partition of $D$ is quasi-uniform then the following inverse inequality holds:

$$
\|\chi\|_{\ell, D} \leq C h^{-1}\|\chi\|_{\ell-1, D}, \quad \ell=1, \ldots, \kappa(r), \quad \forall \chi \in S_{h}^{r, \kappa(r)} .
$$

However, in our analysis, we will not suppose that the above inverse inequality holds unless is clearly stated (see, e.g., Prop. 5.1).

The standard Galerkin finite element approximation $v_{E, h} \in S_{h}^{r, \kappa(r)}$ of the solution $v_{E}$ of (2.11) is specified by requiring

$$
-\Delta_{h} v_{E, h}=P_{h} f
$$

while a finite element approximation $v_{B, h}^{I} \in S_{h}^{r, \kappa(r)}$ of the solution $v_{B}$ of (2.13) is defined by the requirement

$$
\Delta_{h}\left(\Delta_{h} v_{B, h}^{I}\right)=P_{h} f
$$

We introduce, now, the operator $T_{E, h}: \mathrm{L}^{2}(D) \rightarrow S_{h}^{r, \kappa(r)}$ being the solution operator of the finite element method (2.22), i.e. $T_{E, h} f:=v_{E, h}=-\Delta_{h}^{-1} P_{h} f$ for $f \in \mathrm{L}^{2}(D)$, and the operator $T_{B, h}^{I}: \mathrm{L}^{2}(D) \rightarrow S_{h}^{r, \kappa(r)}$ being the solution operator of the finite element method (2.23), i.e. $T_{B, h}^{I} f:=v_{B, h}^{I}=\left(\Delta_{h}\right)^{-2} P_{h} f$ for $f \in \mathrm{L}^{2}(D)$. Hence, the following relation holds

$$
T_{B, h}^{I} f=T_{E, h}\left(T_{E, h} f\right) \quad \forall f \in \mathrm{L}^{2}(D) .
$$

Also, using (2.22), (2.11) and (2.12), we can easily conclude that

$$
\begin{aligned}
\left\|\left(T_{E, h} f\right)^{\prime}\right\|_{0, D} & \leq\left\|\left(T_{E} f\right)^{\prime}\right\|_{0, D} \\
& \leq C\|f\|_{-1, D} \quad \forall f \in \mathrm{L}^{2}(D) .
\end{aligned}
$$

Since $T_{E, h}$ is selfadjoint (see, e.g., Chap. 2 in [24]), i.e., $\left(T_{E, h} f, g\right)_{0, D}=\left(f, T_{E, h} g\right)_{0, D}$ for $f, g \in \mathrm{L}^{2}(D)$, using $(2.24)$ we, easily, arrive at

$$
\left(T_{B, h}^{I} f, g\right)_{0, D}=\left(T_{E, h} f, T_{E, h} g\right)_{0, D} \quad \forall f, g \in \mathrm{L}^{2}(D) .
$$


Due to the approximation property (2.19) of the finite element space $S_{h}^{r, \kappa(r)}$, the theory of the standard Galerkin finite element method for second order elliptic problems (cf., e.g., [5,8]), yields that

$$
\left\|T_{E} f-T_{E, h} f\right\|_{0, D}+h\left\|T_{E} f-T_{E, h} f\right\|_{1, D} \leq C h^{\ell}\left\|T_{E} f\right\|_{\ell, D}, \quad \ell=2, \ldots, r+1, \quad \forall f \in \mathrm{L}^{2}(D) .
$$

Using the estimate above, we derive the following $\mathrm{L}^{2}(D)$-approximation error bounds, for the inverse discrete biharmonic operator $T_{B, h}^{I}$.

Proposition 2.2. Let $r \in\{1,2,3\}$ and $\kappa(r) \in\{1, \ldots, r\}$. Then, it holds that:

$$
\left\|T_{B} f-T_{B, h}^{I} f\right\|_{0, D} \leq C\left\{\begin{array}{lll}
h^{r} & \|f\|_{-1, D}, & r=1,2,3, \\
h^{r+1} & \|f\|_{0, D}, & r=1,2
\end{array} \quad \forall f \in \mathrm{L}^{2}(D)\right.
$$

Proof. See Appendix A.

When $\kappa(r) \geq 2$, we can define another finite element approximation $v_{B, h}^{I I} \in S_{h}^{r, \kappa(r)}$ of the solution $v_{B}$ of (2.13) requiring

$$
B_{h}\left(v_{B, h}^{I I}\right)=P_{h} f
$$

Then, we denote by $T_{B, h}^{I I}: \mathrm{L}^{2}(D) \rightarrow S_{h}^{r, \kappa(r)}$ the corresponding solution operator, i.e. $T_{B, h}^{I I} f:=v_{B, h}^{I I}=B_{h}^{-1} P_{h} f$ for $f \in \mathrm{L}^{2}(D)$, which is selfadjoint, i.e.,

$$
\left(T_{B, h}^{I I} f, g\right)_{0, D}=\left(f, T_{B, h}^{I I} g\right)_{0, D} \quad \forall f, g \in \mathrm{L}^{2}(D)
$$

Also, using (2.13), (1.11), (2.29) and (2.14), we can easily arrive at

$$
\begin{aligned}
\left\|\left(T_{B, h}^{I I} f\right)^{\prime \prime}\right\|_{0, D} & \leq\left\|\left(T_{B} f\right)^{\prime \prime}\right\|_{0, D} \\
& \leq C\|f\|_{-2, D} \quad \forall f \in \mathrm{L}^{2}(D) .
\end{aligned}
$$

Finally, we can provide the inverse biharmonic operator $T_{B, h}^{I I}$, with the following $\mathrm{L}^{2}(D)$-approximation properties.

Proposition 2.3. Let $r \in\{2,3\}$ and $\kappa(r) \geq 2$. Then, it holds that:

$$
\left\|T_{B} f-T_{B, h}^{I I} f\right\|_{0, D} \leq C\left\{\begin{array}{l}
h^{4}\|f\|_{0, D}, \quad r=3, \\
h^{r}\|f\|_{-1, D}, \quad r=2,3
\end{array} \quad \forall f \in \mathrm{L}^{2}(D) .\right.
$$

Proof. See Appendix B.

\section{An estimate FOR THE MOdELling ERror}

In the following theorem, we derive an $\mathrm{L}_{t}^{\infty}\left(\mathrm{L}_{P}^{2}\left(\mathrm{~L}_{x}^{2}\right)\right)$ bound for the modelling error in terms of $\Delta t$ and $\Delta x$.

Theorem 3.1. Let $u$ be the solution of (1.1) and $\widehat{u}$ be the solution of (1.6). Then, there exist a real constant $C>0$, independent of $T, \Delta t$ and $\Delta x$, such that

$$
\max _{[0, T]}\left\{\mathbb{E}\left[\|u-\widehat{u}\|_{0, D}^{2}\right]\right\}^{\frac{1}{2}} \leq C\left[\left(1+(\Delta t)^{\frac{1}{4}}\right)^{\frac{1}{2}} \Delta t^{\frac{3}{8}}+\epsilon^{-\frac{1}{2}} \Delta x^{\frac{3}{2}-\epsilon}\right], \quad \forall \epsilon \in\left(0, \frac{3}{2}\right] .
$$

Proof. Using (1.2), (1.8) and Lemma 2.1, we conclude that

$$
u(t, x)-\widehat{u}(t, x)=\int_{0}^{T} \int_{D}\left[\mathcal{X}_{(0, t)}(s) G(t-s ; x, y)-\widetilde{G}(t, x ; s, y)\right] \mathrm{d} W(s, y) \quad \forall(t, x) \in[0, T] \times \bar{D},
$$


where $\widetilde{G}:(0, T) \times D \rightarrow \mathrm{L}^{2}((0, T) \times D)$ given by

$$
\widetilde{G}(t, x ; s, y):=\frac{1}{\Delta t_{n}} \int_{T_{n}} \mathcal{X}_{(0, t)}\left(s^{\prime}\right)\left(\sum_{i=1}^{2} \widehat{\pi}_{i, j}(y) \int_{D} G\left(t-s^{\prime} ; x, y^{\prime}\right) \widehat{\pi}_{i, j}\left(y^{\prime}\right) \mathrm{d} y^{\prime}\right) \mathrm{d} s^{\prime} \quad \forall(s, y) \in S_{n, j},
$$

for $j=1, \ldots, J_{\Delta}$ and $n=1, \ldots, N_{\Delta}$.

Let $\Theta:=\left\{\mathbb{E}\left[\|u-\widehat{u}\|_{0, D}^{2}\right]\right\}^{\frac{1}{2}}$ and $t \in(0, T]$. Using (3.2) and Itô isometry (2.6), we obtain

$$
\Theta(t)=\left\{\int_{0}^{T} \int_{D} \int_{D}\left[\mathcal{X}_{(0, t)}(s) G(t-s ; x, y)-\widetilde{G}(t, x ; s, y)\right]^{2} \mathrm{~d} x \mathrm{~d} y \mathrm{~d} s\right\}^{\frac{1}{2}} .
$$

Now, we introduce the splitting

$$
\Theta(t) \leq \Theta_{A}(t)+\Theta_{B}(t)
$$

where

$$
\Theta_{A}(t):=\left\{\sum_{n=1}^{N_{\Delta}} \int_{D} \int_{D} \int_{T_{n}}\left[\frac{1}{\Delta t_{n}} \int_{T_{n}} \mathcal{X}_{(0, t)}\left(s^{\prime}\right) G\left(t-s^{\prime} ; x, y\right) \mathrm{d} s^{\prime}-\widetilde{G}(t, x ; s, y)\right]^{2} \mathrm{~d} x \mathrm{~d} y \mathrm{~d} s\right\}^{\frac{1}{2}}
$$

and

$$
\Theta_{B}(t):=\left\{\sum_{n=1}^{N_{\Delta}} \int_{D} \int_{D} \int_{T_{n}}\left[\mathcal{X}_{(0, t)}(s) G(t-s ; x, y)-\frac{1}{\Delta t_{n}} \int_{T_{n}} \mathcal{X}_{(0, t)}\left(s^{\prime}\right) G\left(t-s^{\prime} ; x, y\right) \mathrm{d} s^{\prime}\right]^{2} \mathrm{~d} x \mathrm{~d} y \mathrm{~d} s\right\}^{\frac{1}{2}} .
$$

Estimation of $\Theta_{A}(t)$. Using (1.5) and the $(\cdot, \cdot)_{0, D}$-orthogonality of $\left(\varepsilon_{k}\right)_{k=1}^{\infty}$, we have

$$
\begin{aligned}
\Theta_{A}^{2}(t) & =\sum_{n=1}^{N_{\Delta}} \sum_{j=1}^{J_{\Delta}} \frac{1}{\Delta t_{n}} \int_{D} \int_{D_{j}}\left[\int_{T_{n}} \mathcal{X}_{(0, t)}\left(s^{\prime}\right)\left[G\left(t-s^{\prime} ; x, y\right)-\sum_{i=1}^{2}\left(G\left(t-s^{\prime} ; x, \cdot\right), \widetilde{\pi}_{i, j}(\cdot)\right)_{0, D_{j}} \widetilde{\pi}_{i, j}(y)\right] \mathrm{d} s^{\prime}\right]^{2} \mathrm{~d} y \mathrm{~d} x \\
& =\sum_{n=1}^{N_{\Delta}} \sum_{j=1}^{J_{\Delta}} \frac{1}{\Delta t_{n}}\left[\sum_{k=1}^{\infty}\left(\int_{T_{n}} \mathcal{X}_{(0, t)}\left(s^{\prime}\right) \mathrm{e}^{-2 \lambda_{k}^{4}\left(t-s^{\prime}\right)} \mathrm{d} s^{\prime}\right)^{2} \int_{D_{j}}\left(\varepsilon_{k}(y)-\sum_{i=1}^{2}\left(\varepsilon_{k}, \widetilde{\pi}_{i, j}\right)_{0, D_{j}} \widetilde{\pi}_{i, j}(y)\right)^{2} \mathrm{~d} y\right]
\end{aligned}
$$

from which, using the Cauchy-Schwarz inequality, follows that

$$
\Theta_{A}^{2}(t) \leq \sum_{k=1}^{\infty}\left(\int_{0}^{t} \mathrm{e}^{-4 \lambda_{k}^{4}\left(t-s^{\prime}\right)} \mathrm{d} s^{\prime}\right)\left[\int_{D_{j}}\left(\varepsilon_{k}(y)-\sum_{i=1}^{2}\left(\varepsilon_{k}, \widetilde{\pi}_{i, j}\right)_{0, D_{j}} \widetilde{\pi}_{i, j}(y)\right)^{2} \mathrm{~d} y\right] .
$$

First, we observe that

$$
\int_{0}^{t} \mathrm{e}^{-4 \lambda_{k}^{4}\left(t-s^{\prime}\right)} \mathrm{d} s^{\prime} \leq \frac{1}{4 \lambda_{k}^{4}} \quad \forall k \in \mathbb{N}
$$

Next, by interpolation, using the standard approximation properties of the $\mathrm{L}^{2}\left(D_{j}\right)$-projection operator onto $\mathbb{P}^{1}\left(D_{j}\right)$ (see, e.g. [5]), we have

$$
\begin{aligned}
\left(\int_{D_{j}}\left(\varepsilon_{k}(y)-\sum_{i=1}^{2}\left(\varepsilon_{k}, \tilde{\pi}_{i, j}\right)_{0, D_{j}} \tilde{\pi}_{i, j}(y)\right)^{2} \mathrm{~d} y\right)^{\frac{1}{2}} & \leq C\left(\Delta x_{j}\right)^{2 \theta}\left\|\varepsilon_{k}\right\|_{\dot{\mathrm{H}}^{2 \theta}} \\
& \leq C\left(\Delta x_{j}\right)^{2 \theta} \lambda_{k}^{2 \theta} \quad \forall \theta \in[0,1] .
\end{aligned}
$$


Thus, from (3.4), (3.5) and (3.6) we arrive at

$$
\Theta_{A}(t) \leq C(\Delta x)^{2 \theta}\left(\sum_{k=1}^{\infty} \lambda_{k}^{-\left[1+2\left(\frac{3}{2}-2 \theta\right)\right]}\right)^{\frac{1}{2}} \quad \forall \theta \in\left[0, \frac{3}{4}\right)
$$

Estimation of $\Theta_{B}(t)$. For $t \in(0, T]$, let $\widehat{N}(t):=\min \left\{\ell \in \mathbb{N}: 1 \leq \ell \leq N_{\Delta}\right.$ and $\left.t \leq t_{\ell}\right\}$ and

$$
\widehat{T}_{n}(t):=T_{n} \cap(0, t)=\left\{\begin{array}{ll}
T_{n}, & \text { if } n<\widehat{N}(t) \\
\left(t_{\widehat{N}(t)-1}, t\right), & \text { if } n=\widehat{N}(t)
\end{array}, \quad n=1, \ldots, \widehat{N}(t) .\right.
$$

Thus, using $(1.5)$ and the $(\cdot, \cdot)_{0, D}$-orthogonality of $\left(\varepsilon_{k}\right)_{k=1}^{\infty}$ it follows that

$$
\begin{aligned}
\Theta_{B}^{2}(t) & =\sum_{n=1}^{N_{\Delta}} \frac{1}{\left(\Delta t_{n}\right)^{2}} \int_{D} \int_{D} \int_{T_{n}}\left[\int_{T_{n}}\left[\mathcal{X}_{(0, t)}(s) G(t-s ; x, y)-\mathcal{X}_{(0, t)}\left(s^{\prime}\right) G\left(t-s^{\prime} ; x, y\right)\right] \mathrm{d} s^{\prime}\right]^{2} \mathrm{~d} x \mathrm{~d} y \mathrm{~d} s \\
& =\sum_{n=1}^{N_{\Delta}} \frac{1}{\left(\Delta t_{n}\right)^{2}} \int_{D} \int_{D} \int_{T_{n}}\left[\sum_{k=1}^{\infty} \varepsilon_{k}(x) \varepsilon_{k}(y) \int_{T_{n}}\left[\mathcal{X}_{(0, t)}(s) \mathrm{e}^{-\lambda_{k}^{4}(t-s)}-\mathcal{X}_{(0, t)}\left(s^{\prime}\right) \mathrm{e}^{-\lambda_{k}^{4}\left(t-s^{\prime}\right)}\right] \mathrm{d} s^{\prime}\right]^{2} \mathrm{~d} x \mathrm{~d} y \mathrm{~d} s
\end{aligned}
$$

which yields that

$$
\Theta_{B}^{2}(t) \leq \sum_{k=1}^{\infty} \sum_{n=1}^{\widehat{N}(t)} \frac{1}{\left(\Delta t_{n}\right)^{2}} \Psi_{n}^{k}(t)
$$

where

$$
\Psi_{n}^{k}(t):=\int_{T_{n}}\left[\int_{T_{n}}\left(\mathcal{X}_{(0, t)}(s) \mathrm{e}^{-\lambda_{k}^{4}(t-s)}-\mathcal{X}_{(0, t)}\left(s^{\prime}\right) \mathrm{e}^{-\lambda_{k}^{4}\left(t-s^{\prime}\right)}\right) \mathrm{d} s^{\prime}\right]^{2} \mathrm{~d} s .
$$

Let $k \in \mathbb{N}$ and $n \in\{1, \ldots, \widehat{N}(t)-1\}$. Then, we have

$$
\begin{aligned}
\Psi_{n}^{k}(t) & =\int_{T_{n}}\left(\int_{T_{n}} \int_{s}^{s^{\prime}} \lambda_{k}^{4} \mathrm{e}^{-\lambda_{k}^{4}(t-\tau)} \mathrm{d} \tau \mathrm{d} s^{\prime}\right)^{2} \mathrm{~d} s \\
& \leq \int_{T_{n}}\left(\int_{T_{n}} \int_{t_{n-1}}^{\max \left\{s^{\prime}, s\right\}} \lambda_{k}^{4} \mathrm{e}^{-\lambda_{k}^{4}(t-\tau)} \mathrm{d} \tau \mathrm{d} s^{\prime}\right)^{2} \mathrm{~d} s \\
& \leq 2 \int_{T_{n}}\left(\int_{T_{n}} \int_{t_{n-1}}^{s^{\prime}} \lambda_{k}^{4} \mathrm{e}^{-\lambda_{k}^{4}(t-\tau)} \mathrm{d} \tau \mathrm{d} s^{\prime}\right)^{2} \mathrm{~d} s+2 \int_{T_{n}}\left(\int_{T_{n}} \int_{t_{n-1}}^{s} \lambda_{k}^{4} \mathrm{e}^{-\lambda_{k}^{4}(t-\tau)} \mathrm{d} \tau \mathrm{d} s^{\prime}\right)^{2} \mathrm{~d} s \\
& \leq 2 \Delta t_{n}\left(\int_{T_{n}} \int_{t_{n-1}}^{s^{\prime}} \lambda_{k}^{4} \mathrm{e}^{-\lambda_{k}^{4}(t-\tau)} \mathrm{d} \tau \mathrm{d} s^{\prime}\right)^{2}+2\left(\Delta t_{n}\right)^{2} \int_{T_{n}}\left(\int_{t_{n-1}}^{s} \lambda_{k}^{4} \mathrm{e}^{-\lambda_{k}^{4}(t-\tau)} \mathrm{d} \tau\right)^{2} \mathrm{~d} s
\end{aligned}
$$

from which, using the Cauchy-Schwarz inequality and integrating by parts, we obtain

$$
\begin{aligned}
\Psi_{n}^{k}(t) & \leq 4\left(\Delta t_{n}\right)^{2} \int_{T_{n}}\left(\mathrm{e}^{-\lambda_{k}^{4}(t-s)}-\mathrm{e}^{-\lambda_{k}^{4}\left(t-t_{n-1}\right)}\right)^{2} \mathrm{~d} s \\
& \leq 4\left(\Delta t_{n}\right)^{2}\left(1-\mathrm{e}^{-\lambda_{k}^{4} \Delta t}\right)^{2} \int_{T_{n}} \mathrm{e}^{-2 \lambda_{k}^{4}(t-s)} \mathrm{d} s \\
& \leq 2\left(\Delta t_{n}\right)^{2}\left(1-\mathrm{e}^{-\lambda_{k}^{4} \Delta t}\right)^{2} \frac{\mathrm{e}^{-2 \lambda_{k}^{4}\left(t-t_{n}\right)}-\mathrm{e}^{-2 \lambda_{k}^{4}\left(t-t_{n-1}\right)}}{\lambda_{k}^{4}}
\end{aligned}
$$


Thus, by summing with respect to $n$, we obtain

$$
\sum_{n=1}^{\widehat{N}(t)-1} \frac{1}{\left(\Delta t_{n}\right)^{2}} \Psi_{n}^{k}(t) \leq 2 \frac{\left(1-\mathrm{e}^{-\lambda_{k}^{4} \Delta t}\right)^{2}}{\lambda_{k}^{4}} .
$$

Considering, now, the case $n=\widehat{N}(t)$, we have

$$
\Psi_{\widehat{N}(t)}^{k}(t)=\Psi_{A}^{k}(t)+\Psi_{B}^{k}(t)
$$

with

$$
\begin{aligned}
& \Psi_{A}^{k}(t):=\int_{\widehat{T}_{\widehat{N}(t)}(t)}\left(\int_{t_{\widehat{N}(t)-1}}^{t} \int_{s^{\prime}}^{s} \lambda_{k}^{4} \mathrm{e}^{-\lambda_{k}^{4}(t-\tau)} \mathrm{d} \tau \mathrm{d} s^{\prime}+\int_{t}^{t_{\widehat{N}(t)}} \mathrm{e}^{-\lambda_{k}^{4}(t-s)} \mathrm{d} s^{\prime}\right)^{2} \mathrm{~d} s \\
& \Psi_{B}^{k}(t):=\int_{t}^{t}\left(\int_{t_{\widehat{N}(t)}(t)}^{t} \mathrm{e}^{-\lambda_{k}^{4}\left(t-s^{\prime}\right)} \mathrm{d} s^{\prime}\right)^{2} \mathrm{~d} s .
\end{aligned}
$$

Then, we have

$$
\begin{aligned}
\Psi_{B}^{k}(t) & \leq \frac{\Delta t_{\widehat{N}(t)}}{\lambda_{k}^{8}}\left[1-\mathrm{e}^{-\lambda_{k}^{4}\left(t-t_{\widehat{N}(t)-1}\right)}\right]^{2} \\
& \leq \frac{\Delta t_{\widehat{N}(t)}}{\lambda_{k}^{8}}\left(1-\mathrm{e}^{-\lambda_{k}^{4} \Delta t_{\widehat{N}(t)}}\right)^{2}
\end{aligned}
$$

and

$$
\begin{aligned}
\Psi_{A}^{k}(t) & \leq \int_{t_{\widehat{N}(t)-1}}^{t}\left[\int_{t_{\widehat{N}(t)-1}}^{t} \int_{s^{\prime}}^{s} \lambda_{k}^{4} \mathrm{e}^{-\lambda_{k}^{4}(t-\tau)} \mathrm{d} \tau \mathrm{d} s^{\prime}+\Delta t_{\widehat{N}(t)} \mathrm{e}^{-\lambda_{k}^{4}(t-s)}\right]^{2} \mathrm{~d} s \\
& \leq 2 \int_{t_{\widehat{N}(t)-1}}^{t}\left[\int_{t_{\widehat{N}(t)-1}}^{t} \int_{s^{\prime}}^{s} \lambda_{k}^{4} \mathrm{e}^{-\lambda_{k}^{4}(t-\tau)} \mathrm{d} \tau \mathrm{d} s^{\prime}\right]^{2} \mathrm{~d} s+\frac{\left(\Delta t_{\widehat{N}(t)}\right)^{2}}{\lambda_{k}^{4}}\left[1-\mathrm{e}^{\left.-2 \lambda_{k}^{4}\left(t-t_{\widehat{N}(t)-1}\right)\right]}\right. \\
& \leq 2 \int_{t_{\widehat{N}(t)-1}}^{t}\left[\int_{t_{\widehat{N}(t)-1}}^{t} \int_{t_{\widehat{N}(t)-1}}^{\max \left\{s, s^{\prime}\right\}} \lambda_{k}^{4} \mathrm{e}^{-\lambda_{k}^{4}(t-\tau)} \mathrm{d} \tau \mathrm{d} s^{\prime}\right]^{2} \mathrm{~d} s+\frac{\left(\Delta t_{\widehat{N}(t)}\right)^{2}}{\lambda_{k}^{4}}\left(1-\mathrm{e}^{\left.-2 \lambda_{k}^{4} \Delta t_{\widehat{N}(t)}\right)}\right. \\
& \leq 8\left(\Delta t_{\widehat{N}(t)}\right)^{2} \int_{t_{\widehat{N}(t)-1}}^{t}\left[\int_{t_{\widehat{N}(t)-1}}^{s} \lambda_{k}^{4} \mathrm{e}^{-\lambda_{k}^{4}(t-\tau)} \mathrm{d} \tau\right]^{2} \mathrm{~d} s+\frac{\left(\Delta t_{\widehat{N}(t)}\right)^{2}}{\lambda_{k}^{4}}\left(1-\mathrm{e}^{\left.-2 \lambda_{k}^{4} \Delta t_{\widehat{N}(t)}\right)}\right. \\
& \leq 8\left(\Delta t_{\widehat{N}(t)}\right)^{2} \int_{t_{\widehat{N}(t)-1}}^{t}\left[\mathrm{e}^{-\lambda_{k}^{4}(t-s)}-\mathrm{e}^{-\lambda_{k}^{4}\left(t-t_{\widehat{N}(t)-1}\right)}\right]^{2} \mathrm{~d} s+\frac{\left(\Delta t_{\widehat{N}(t)}\right)^{2}}{\lambda_{k}^{4}}\left(1-\mathrm{e}^{-2 \lambda_{k}^{4} \Delta t_{\widehat{N}(t)}}\right),
\end{aligned}
$$

which, along with (3.10), gives

$$
\Psi_{\widehat{N}(t)}^{k} \leq 5 \frac{\left(\Delta t_{\widehat{N}(t)}\right)^{2}}{\lambda_{k}^{4}}\left(1-\mathrm{e}^{-2 \lambda_{k}^{4} \Delta t_{\widehat{N}(t)}}\right)+\frac{\Delta t_{\widehat{N}(t)}}{\lambda_{k}^{8}}\left(1-\mathrm{e}^{-\lambda_{k}^{4} \Delta t_{\widehat{N}(t)}}\right)^{2} .
$$

Since the mean value theorem yields: $1-\mathrm{e}^{-\lambda_{k}^{4} \Delta t_{\widehat{N}(t)}} \leq \lambda_{k}^{4} \Delta t_{\widehat{N}(t)}$, the above inequality takes the form

$$
\frac{1}{\left(\Delta t_{\widehat{N}(t)}\right)^{2}} \Psi_{\widehat{N}(t)}^{k} \leq 6 \frac{1-\mathrm{e}^{-2 \lambda_{k}^{4} \Delta t} \widehat{N_{N}(t)}}{\lambda_{k}^{4}} .
$$


Combining (3.8), (3.9) and (3.11) we obtain

$$
\Theta_{B}^{2}(t) \leq 8 \sum_{k=1}^{\infty} \frac{1-\mathrm{e}^{-2 \lambda_{k}^{4} \Delta t}}{\lambda_{k}^{4}}
$$

To get a convergence estimate we have to exploit the way the series depends on $\Delta t$ in the above relation:

$$
\begin{aligned}
\sum_{k=1}^{\infty} \frac{1-\mathrm{e}^{-2 \lambda_{k}^{4} \Delta t}}{\lambda_{k}^{4}} & \leq \frac{1-\mathrm{e}^{-2 \pi^{4} \Delta t}}{\pi^{4}}+\int_{1}^{\infty} \frac{1-\mathrm{e}^{-2 x^{4} \pi^{4} \Delta t}}{x^{4} \pi^{4}} \mathrm{~d} x \\
& \leq \frac{4}{3 \pi^{4}}\left(1-\mathrm{e}^{-2 \pi^{4} \Delta t}\right)+\frac{8 \Delta t}{3} \int_{1}^{\infty} \mathrm{e}^{-2 x^{4} \pi^{4} \Delta t} \mathrm{~d} x \\
& \leq \frac{8}{3} \Delta t+\frac{8}{3 \pi}(\Delta t)^{\frac{3}{4}} \int_{0}^{\infty} \mathrm{e}^{-2 y^{4}} \mathrm{~d} y \\
& \leq\left[\frac{8}{3}(\Delta t)^{\frac{1}{4}}+\frac{28}{9 \pi}\right](\Delta t)^{\frac{3}{4}}
\end{aligned}
$$

Using the bounds (3.12) and (3.13) we arrive at

$$
\Theta_{B}(t) \leq 8\left[\frac{1}{3}(\Delta t)^{\frac{1}{4}}+\frac{7}{18 \pi}\right]^{\frac{1}{2}}(\Delta t)^{\frac{3}{8}} .
$$

The error bound (3.1) follows by observing that $\Theta(0)=0$ and combining the bounds (3.3), (3.7), (2.7) and (3.14).

Remark 3.2. In contrast to (1.7), the discrete space-time white noise kernel $\widehat{W}$ proposed in [1] is piecewise constant in space and has the form: $\widehat{W}(t, x):=\frac{1}{\Delta t \Delta x} \sum_{n=1}^{N_{\Delta}} \sum_{j=1}^{J_{\Delta}} \mathcal{X}_{S_{n, j}}(t, x) R_{n, j}$ for all $(t, x) \in[0, T] \times \bar{D}$, where $R_{n, j}:=\int_{S_{n, j}} 1 \mathrm{~d} W(t, x)$. In [20], we show that the use of the above piecewise constant in space and time discrete space-time white noise yields a different regularized problem with corresponding modelling error $O\left(\Delta t^{\frac{3}{8}}+\Delta x\right)$ which is of lower order with respect to $\Delta x$.

\section{Time-Discrete APPROXimations}

In this section we consider the Backward Euler time-discretization method for problem (1.6). Assuming that the partition of the time interval is uniform, we derive a discrete in time $\mathrm{L}_{t}^{\infty}\left(\mathrm{L}_{P}^{2}\left(\mathrm{~L}_{x}^{2}\right)\right)$ error estimate with constants independent of $N_{\Delta}$ and $J_{\Delta}$ (see Thm. 4.2). We are interested in deriving such an error estimate for the Backward Euler time-discrete approximations in order to use it later to estimate the approximation error of the Backward Euler fully-discrete approximations in a discrete in time $\mathrm{L}_{t}^{\infty}\left(\mathrm{L}_{P}^{2}\left(\mathrm{~L}_{x}^{2}\right)\right.$ ) norm (see Thm. 6.9).

The Backward Euler time-discretization method of the problem (1.6) constructs, for $m=0, \ldots, M$, an approximation $\widehat{U}^{m}$ of $\widehat{u}\left(\tau_{m}, \cdot\right)$ following the steps below:

Step TD1. Set

$$
\widehat{U}^{0}:=0 .
$$

Step TD2. For $m=1, \ldots, M$, find $\widehat{U}^{m} \in \dot{\mathbf{H}}^{4}(D)$ such that

$$
\widehat{U}^{m}-\widehat{U}^{m-1}+k_{m} \partial_{x}^{4} \widehat{U}^{m}=\int_{\Delta_{m}} \widehat{W} \mathrm{~d} s \quad \text { a.s. }
$$

To proceed to an error estimate in a discrete in time $\mathrm{L}_{t}^{\infty}\left(\mathrm{L}_{P}^{2}\left(\mathrm{~L}_{x}^{2}\right)\right)$ norm, we will, first, derive, using interpolation, a low regularity a priori error estimate in a discrete in time $\mathrm{L}_{t}^{2}\left(\mathrm{~L}_{x}^{2}\right)$ for the Backward Euler time-discrete 
approximations, $\left(W^{m}\right)_{m=0}^{M}$, of the solution $w$ to the deterministic problem $(1.3)(c f .[23,26,27])$, which are defined below:

Step DTD1. Set

$$
W^{0}:=w_{0}
$$

Step DTD2. For $m=1, \ldots, M$, find $W^{m} \in \dot{\mathbf{H}}^{4}(D)$ such that

$$
W^{m}-W^{m-1}+k_{m} \partial_{x}^{4} W^{m}=0 .
$$

Proposition 4.1. Let $\left(W^{m}\right)_{m=0}^{M}$ be the Backward Euler time-discrete approximations of the solution $w$ of the problem (1.3) defined in (4.3)-(4.4). If $w_{0} \in \dot{\mathbf{H}}^{2}(D)$, then, there exists a constant $C>0$, independent of $T, \Delta t$, $\Delta x, M$ and $\left(k_{m}\right)_{m=1}^{M}$, such that

$$
\left(\sum_{m=1}^{M} k_{m}\left\|W^{m}-w\left(\tau_{m}, \cdot\right)\right\|_{0, D}^{2}\right)^{\frac{1}{2}} \leq C\left(k_{\max }\right)^{\theta}\left\|w_{0}\right\|_{\dot{\mathrm{H}}^{4 \theta-2}} \quad \forall \theta \in[0,1] .
$$

Proof. The estimate (4.5) follows by interpolation, after proving it for $\theta=1$ and $\theta=0$.

Let $E^{m}(\cdot):=w\left(\tau_{m}, \cdot\right)-W^{m}(\cdot)$ for $m=0, \ldots, M$, and $\rho_{m}:=\int_{\Delta_{m}}\left(w\left(\tau_{m}, \cdot\right)-w(\tau, \cdot)\right) \mathrm{d} \tau$ for $m=1, \ldots, M$. First, we use (1.3) and (4.4), to conclude that $T_{B}\left(E^{m}-E^{m-1}\right)+k_{m} E^{m}=\rho_{m}$ for $m=1, \ldots, M$. Then, we take the $\mathrm{L}^{2}(D)$-inner product of both sides of the latter relation with $E^{m}$, use (2.16), and then sum with respect to $m$ from 1 up to $M$, to obtain

$$
\sum_{m=1}^{M}\left(T_{E} E^{m}-T_{E} E^{m-1}, T_{E} E^{m}\right)_{0, D}+\sum_{m=1}^{M} k_{m}\left\|E^{m}\right\|_{0, D}^{2}=\sum_{m=1}^{M}\left(\rho_{m}, E^{m}\right)_{0, D} .
$$

Since $E^{0}=0$ and $\left(T_{E} E^{m}-T_{E} E^{m-1}, T_{E} E^{m}\right)_{0, D} \geq \frac{1}{2}\left(\left\|T_{E} E^{m}\right\|_{0, D}^{2}-\left\|T_{E} E^{m-1}\right\|_{0, D}^{2}\right)$ for $m=1, \ldots, M$, from (4.6), we arrive at

$$
\sum_{m=1}^{M} k_{m}\left\|E^{m}\right\|_{0, D}^{2} \leq \sum_{m=1}^{M} k_{m}^{-1}\left\|\rho_{m}\right\|_{0, D}^{2}
$$

Next, we use the Cauchy-Schwarz inequality to bound $\rho_{m}$ as follows:

$$
\begin{aligned}
\left\|\rho_{m}\right\|_{0, D}^{2} & =\int_{D}\left(\int_{\Delta_{m}} \int_{\tau}^{\tau_{m}} \partial_{\tau} w(s, x) \mathrm{d} s \mathrm{~d} \tau\right)^{2} \mathrm{~d} x \\
& \leq \int_{D}\left(\int_{\Delta_{m}} \int_{\Delta_{m}}\left|\partial_{\tau} w(s, x)\right| \mathrm{d} s \mathrm{~d} \tau\right)^{2} \mathrm{~d} x \\
& \leq\left(k_{m}\right)^{3} \int_{\Delta_{m}}\left\|\partial_{\tau} w(s, \cdot)\right\|_{0, D}^{2} \mathrm{~d} s, \quad m=1, \ldots, M .
\end{aligned}
$$

Now, we combine (4.7), (4.8) and (2.18) (with $\beta=0, \ell=1, p=0$ ) to obtain

$$
\begin{aligned}
\sum_{m=1}^{M} k_{m}\left\|E^{m}\right\|_{0, D}^{2} & \leq\left(k_{\max }\right)^{2} \int_{0}^{T}\left\|\partial_{\tau} w(s, \cdot)\right\|_{0, D}^{2} \mathrm{~d} s \\
& \leq C\left(k_{\max }\right)^{2}\left\|w_{0}\right\|_{\dot{\mathbf{H}}^{2}}^{2},
\end{aligned}
$$

which yields (4.5) for $\theta=1$.

Since (4.4) is equivalent to $T_{B}\left(W^{m}-W^{m-1}\right)+k_{m} W^{m}=0$ for $m=1, \ldots, M$, we take the $\mathrm{L}^{2}(D)$-inner product of both sides of it with $W^{m}$ and use (2.16), to obtain $\left(T_{E} W^{m}-T_{E} W^{m-1}, T_{E} W^{m}\right)_{0, D}+k_{m}\left\|W^{m}\right\|_{0, D}^{2}=0$ 
for $m=1, \ldots, M$. Proceeding as above it follows that $\left\|T_{E} W^{m}\right\|_{0, D}^{2}-\left\|T_{E} W^{m-1}\right\|_{0, D}^{2}+2 k_{m}\left\|W^{m}\right\|_{o, D}^{2} \leq 0$ for $m=1, \ldots, M$. Then, summing with respect to $m$ from 1 up to $M$, and using (2.12) and (2.4) we obtain

$$
\begin{aligned}
\sum_{k=1}^{M} k_{m}\left\|W^{m}\right\|_{0, D}^{2} & \leq \frac{1}{2}\left\|T_{E} w^{0}\right\|_{0, D}^{2} \\
& \leq C\left\|w_{0}\right\|_{\mathbf{H}^{-2}}^{2} .
\end{aligned}
$$

In addition we have

$$
\begin{aligned}
\sum_{m=1}^{M} k_{m}\left\|w\left(\tau_{m}, \cdot\right)\right\|_{0, D}^{2} & \leq \sum_{m=1}^{M} \int_{D}\left(\int_{\Delta_{m}} \partial_{\tau}\left[\left(\tau-\tau_{m-1}\right) w^{2}(\tau, x)\right] \mathrm{d} \tau\right) \mathrm{d} x \\
& \leq \sum_{m=1}^{M} \int_{D}\left(\int_{\Delta_{m}}\left[w^{2}(\tau, x)+2\left(\tau-\tau_{m-1}\right) w_{\tau}(\tau, x) w(\tau, x)\right] \mathrm{d} \tau\right) \mathrm{d} x \\
& \leq \sum_{m=1}^{M} \int_{\Delta_{m}}\left[2\|w(\tau, \cdot)\|_{0, D}^{2}+\left(\tau-\tau_{m-1}\right)^{2}\left\|w_{\tau}(\tau, \cdot)\right\|_{0, D}^{2}\right] \mathrm{d} \tau \\
& \leq 2 \int_{0}^{T}\left[\|w(\tau, \cdot)\|_{0, D}^{2}+\tau^{2}\left\|w_{\tau}(\tau, \cdot)\right\|_{0, D}^{2}\right] \mathrm{d} \tau,
\end{aligned}
$$

which, along with (2.18) (taking $(\beta, \ell, p)=(0,0,0)$ and $(\beta, \ell, p)=(2,1,0))$ and $(2.4)$, yields

$$
\sum_{m=1}^{M} k_{m}\left\|w\left(\tau_{m}, \cdot\right)\right\|_{0, D}^{2} \leq C\left\|w_{0}\right\|_{\mathrm{H}^{-2}}^{2}
$$

Thus, the estimate (4.5) for $\theta=0$ follows easily combining (4.10) and (4.11).

Next theorem proves a discrete in time $\mathrm{L}_{t}^{\infty}\left(\mathrm{L}_{P}^{2}\left(\mathrm{~L}_{x}^{2}\right)\right)$ convergence estimate for the Backward Euler time discrete approximations of $\widehat{u}$, over a uniform partition of $[0, T]$.

Theorem 4.2. Let $\widehat{u}$ be the solution of (1.6) and $\left(\widehat{U}^{m}\right)_{m=0}^{M}$ be the Backward Euler time-discrete approximations specified in (4.1)-(4.2). If $k_{m}=\Delta \tau$ for $m=1, \ldots, M$, then there exists a constant $C>0$, independent of $T$, $\Delta t, \Delta x$ and $\Delta \tau$, such that

$$
\max _{1 \leq m \leq M}\left\{\mathbb{E}\left[\left\|\widehat{U}^{m}-\widehat{u}\left(\tau_{m}, \cdot\right)\right\|_{0, D}^{2}\right]\right\}^{\frac{1}{2}} \leq C \widetilde{\omega}(\Delta \tau, \epsilon) \Delta \tau^{\frac{3}{8}-\epsilon}, \quad \forall \epsilon \in\left(0, \frac{3}{8}\right]
$$

where $\widetilde{\omega}(\Delta \tau, \epsilon):=\epsilon^{-\frac{1}{2}}+(\Delta \tau)^{\epsilon}\left(1+(\Delta \tau)^{\frac{1}{4}}\right)^{\frac{1}{2}}$.

Proof. Let $I: \mathrm{L}^{2}(D) \rightarrow \mathrm{L}^{2}(D)$ be the identity operator, $\Lambda: \mathrm{L}^{2}(D) \rightarrow \dot{\mathbf{H}}^{4}(D)$ be the inverse elliptic operator $\Lambda:=\left(I+\Delta \tau \partial_{x}^{4}\right)^{-1}$ which has Green function $G_{\Lambda}(x, y)=\sum_{k=1}^{\infty} \frac{\varepsilon_{k}(x) \varepsilon_{k}(y)}{1+\Delta \tau \lambda_{k}^{4}}$, i.e. $\Lambda f(x)=\int_{D} G_{\Lambda}(x, y) f(y) \mathrm{d} y$ for $x \in \bar{D}$ and $f \in \mathrm{L}^{2}(D)$. Obviously, $G_{\Lambda}(x, y)=G_{\Lambda}(y, x)$ for $x, y \in D$, and $G_{\Lambda} \in \mathrm{L}^{2}(D \times D)$. Also, for $m \in \mathbb{N}$, we denote by $G_{\Lambda, m}$ the Green function of $\Lambda^{m}$, i.e. $G_{\Lambda, m}=\sum_{k=1}^{\infty} \frac{\varepsilon_{k}(x) \varepsilon_{k}(y)}{\left(1+\Delta \tau \lambda_{k}^{4}\right)^{m}}$. Thus, from (4.2), using an induction argument, we conclude that $\widehat{U}^{m}=\sum_{j=1}^{m} \int_{\Delta_{j}} \Lambda^{m-j+1} \widehat{W}(\tau, \cdot) \mathrm{d} \tau$ for $m=1, \ldots, M$, which is written, equivalently, as follows:

$$
\widehat{U}^{m}(x)=\int_{0}^{\tau_{m}} \int_{D} \widehat{\mathcal{K}}_{m}(\tau ; x, y) \widehat{W}(\tau, y) \mathrm{d} y \mathrm{~d} \tau \quad \forall x \in \bar{D}, \quad m=1, \ldots, M,
$$

where $\widehat{\mathcal{K}}_{m}(\tau ; x, y):=\sum_{j=1}^{m} \mathcal{X}_{\Delta_{j}}(\tau) G_{\Lambda, m-j+1}(x, y) \quad \forall \tau \in[0, T], \quad \forall x, y \in D$. 
Let $m \in\{1, \ldots, M\}$ and $\mathcal{E}^{m}:=\mathbb{E}\left[\left\|\widehat{U}^{m}-\widehat{u}\left(\tau_{m}, \cdot\right)\right\|_{0, D}^{2}\right]$. First, we use (4.13), (1.8), (2.10), (2.6), (2.5) and (2.9), to obtain

$$
\begin{aligned}
\mathcal{E}^{m} & =\mathbb{E}\left[\int_{D}\left(\int_{0}^{T} \int_{D} \mathcal{X}_{\left(0, \tau_{m}\right)}(\tau)\left[\widehat{\mathcal{K}}_{m}(\tau ; x, y)-G\left(\tau_{m}-\tau ; x, y\right)\right] \widehat{W}(\tau, y) \mathrm{d} y \mathrm{~d} \tau\right)^{2} \mathrm{~d} x\right] \\
& \leq \int_{0}^{\tau_{m}}\left(\int_{D} \int_{D}\left[\widehat{\mathcal{K}}_{m}(\tau ; x, y)-G\left(\tau_{m}-\tau ; x, y\right)\right]^{2} \mathrm{~d} y \mathrm{~d} x\right) \mathrm{d} \tau \\
& \leq \sum_{\ell=1}^{m} \int_{\Delta_{\ell}}\left(\int_{D} \int_{D}\left[G_{\Lambda, m-\ell+1}(x, y)-G\left(\tau_{m}-\tau ; x, y\right)\right]^{2} \mathrm{~d} y \mathrm{~d} x\right) \mathrm{d} \tau \\
& \leq \sum_{\ell=1}^{m} \int_{\Delta_{\ell}}\left\|\Lambda^{m-\ell+1}-\mathcal{S}\left(\tau_{m}-\tau\right)\right\|_{\mathrm{HS}}^{2} \mathrm{~d} \tau .
\end{aligned}
$$

Now, we introduce the splitting

where

$$
\mathcal{E}^{m} \leq \mathcal{B}_{1}^{m}+\mathcal{B}_{2}^{m}
$$

$$
\begin{aligned}
& \mathcal{B}_{1}^{m}:=2 \sum_{\ell=1}^{m} \int_{\Delta_{\ell}}\left\|\Lambda^{m-\ell+1}-\mathcal{S}\left(\tau_{m}-\tau_{\ell-1}\right)\right\|_{\mathrm{HS}}^{2} \mathrm{~d} \tau, \\
& \mathcal{B}_{2}^{m}:=2 \sum_{\ell=1}^{m} \int_{\Delta_{\ell}}\left\|\mathcal{S}\left(\tau_{m}-\tau_{\ell-1}\right)-\mathcal{S}\left(\tau_{m}-\tau\right)\right\|_{\mathrm{HS}}^{2} \mathrm{~d} \tau .
\end{aligned}
$$

Estimation of $\mathcal{B}_{1}^{m}$. By the definition of the Hilbert-Schmidt norm, we have

$$
\begin{aligned}
\mathcal{B}_{1}^{m} & \leq 2 \Delta \tau \sum_{\ell=1}^{m}\left(\sum_{k=1}^{\infty}\left\|\Lambda^{m-\ell+1} \varepsilon_{k}-\mathcal{S}\left(\tau_{m}-\tau_{\ell-1}\right) \varepsilon_{k}\right\|_{0, D}^{2}\right) \\
& \leq 2 \sum_{k=1}^{\infty}\left(\sum_{\ell=1}^{m} \Delta \tau\left\|\Lambda^{m-\ell+1} \varepsilon_{k}-\mathcal{S}\left(\tau_{m}-\tau_{\ell-1}\right) \varepsilon_{k}\right\|_{0, D}^{2}\right) \\
& \leq 2 \sum_{k=1}^{\infty}\left(\sum_{\ell=1}^{m} \Delta \tau\left\|\Lambda^{\ell} \varepsilon_{k}-\mathcal{S}\left(\tau_{\ell}\right) \varepsilon_{k}\right\|_{0, D}^{2}\right)
\end{aligned}
$$

Let $\theta \in\left[0, \frac{3}{8}\right)$. Using the deterministic error estimate (4.5), we obtain

$$
\begin{aligned}
\mathcal{B}_{1}^{m} & \leq C(\Delta \tau)^{2 \theta} \sum_{k=1}^{\infty}\left\|\varepsilon_{k}\right\|_{\mathbf{H}^{4 \theta-2}}^{2} \\
& \leq C(\Delta \tau)^{2 \theta} \sum_{k=1}^{\infty} \frac{1}{\lambda_{k}^{1+8\left(\frac{3}{8}-\theta\right)}} .
\end{aligned}
$$

The convergence of the series is ensured because $\frac{3}{8}-\theta>0$.

Estimation of $\mathcal{B}_{2}^{m}$. Using, again, the definition of the Hilbert-Schmidt norm we have

$$
\mathcal{B}_{2}^{m}=2 \sum_{k=1}^{\infty}\left\{\sum_{\ell=1}^{m} \int_{\Delta_{\ell}}\left\|\mathcal{S}\left(\tau_{m}-\tau_{\ell-1}\right) \varepsilon_{k}-\mathcal{S}\left(\tau_{m}-\tau\right) \varepsilon_{k}\right\|_{0, D}^{2} \mathrm{~d} \tau\right\} .
$$


Observing that $\mathcal{S}(t) \varepsilon_{k}=\mathrm{e}^{-\lambda_{k}^{4} t} \varepsilon_{k}$ for $t \geq 0,(4.16)$ yields

$$
\begin{aligned}
\mathcal{B}_{2}^{m} & =2 \sum_{k=1}^{\infty}\left[\sum_{\ell=1}^{m} \int_{\Delta_{\ell}}\left(\int_{D}\left[\mathrm{e}^{-\lambda_{k}^{4}\left(\tau_{m}-\tau_{\ell-1}\right)}-\mathrm{e}^{-\lambda_{k}^{4}\left(\tau_{m}-\tau\right)}\right]^{2} \varepsilon_{k}^{2}(x) \mathrm{d} x\right) \mathrm{d} \tau\right] \\
& =2 \sum_{k=1}^{\infty}\left[\sum_{\ell=1}^{m} \int_{\Delta_{\ell}} \mathrm{e}^{-2 \lambda_{k}^{4}\left(\tau_{m}-\tau\right)}\left[1-\mathrm{e}^{-\lambda_{k}^{4}\left(\tau-\tau_{\ell-1}\right)}\right]^{2} \mathrm{~d} \tau\right] \\
& \leq 2 \sum_{k=1}^{\infty}\left(1-\mathrm{e}^{-\lambda_{k}^{4} \Delta \tau}\right)^{2}\left[\int_{0}^{\tau_{m}} \mathrm{e}^{-2 \lambda_{k}^{4}\left(\tau_{m}-\tau\right)} \mathrm{d} \tau\right] \\
& \leq \sum_{k=1}^{\infty} \frac{1-\mathrm{e}^{-2 \lambda_{k}^{4} \Delta \tau}}{\lambda_{k}^{4}}
\end{aligned}
$$

from which, applying (3.13), we obtain

$$
\mathcal{B}_{2}^{m} \leq C\left(1+(\Delta \tau)^{\frac{1}{4}}\right)(\Delta \tau)^{\frac{3}{4}}
$$

The estimate (4.12) follows by (4.14), (4.15), (4.17) and (2.7).

\section{SpaCe-Discrete approximations}

Here, we consider a finite element space-discretization of the solution of (1.6), for which we derive an $\mathrm{L}_{t}^{\infty}\left(\mathrm{L}_{P}^{2}\left(\mathrm{~L}_{x}^{2}\right)\right.$ ) error estimate with constants independent of $N_{\Delta}$ and $J_{\Delta}$ (see Thm. 5.3). This is only a preliminary step in deriving a discrete in time $\mathrm{L}_{t}^{2}\left(\mathrm{~L}_{P}^{2}\left(\mathrm{~L}_{x}^{2}\right)\right)$ error estimate for the Backward Euler fully-discrete approximations of $\widehat{u}$ (see Thm. 6.3).

Let $r \in\{1,2,3\}$ and $\kappa(r) \in\{1, \ldots, r\}$. The space-discrete approximation of the solution $\widehat{u}$ of $(1.6)$ is the solution of the following initial value problem: find a stochastic function $\widehat{u}_{h}:[0, T] \rightarrow S_{h}^{r, \kappa(r)}$ such that

$$
\begin{aligned}
\partial_{t} \widehat{u}_{h}+Q_{h} \widehat{u}_{h}=P_{h} \widehat{W} \quad \text { on }(0, T], \quad \text { a.s. } \\
\widehat{u}_{h}(0)=0
\end{aligned}
$$

where $Q_{h}: S_{h}^{r, \kappa(r)} \rightarrow S_{h}^{r, \kappa(r)}$ given by $Q_{h}=\Delta_{h}^{2}$ when $\kappa(r) \geq 1$, or, $Q_{h}=B_{h}$ when $\kappa(r) \geq 2$.

As a first step towards obtaining an $\mathrm{L}_{t}^{\infty}\left(\mathrm{L}_{P}^{2}\left(\mathrm{~L}_{x}^{2}\right)\right)$ convergence estimate for the space-discrete approximation $\widehat{u}_{h}$, we will derive a low regularity $\mathrm{L}_{t}^{2}\left(\mathrm{~L}_{x}^{2}\right)$ error estimate for the space-discrete approximation $w_{h}$ of the solution $w$ of (1.3) (cf. $[3,26])$, which is defined as the solution of the following initial value problem: find $w_{h}:[0, T] \rightarrow S_{h}^{r, \kappa(r)}$ such that

$$
\begin{gathered}
\partial_{t} w_{h}+Q_{h} w_{h}=0 \quad \text { on }(0, T], \\
w_{h}(0)=P_{h} w_{0} .
\end{gathered}
$$

Since $w_{h}$ can be considered as the value of a linear operator of the initial condition $w_{0}$, we will write it as $w_{h}(t, \cdot)=\left[\mathcal{S}_{h}(t) w_{0}\right](\cdot)$ for $t \in[0, T]$. Thus, by Duhamel's principle $(c f .[24])$, we have

$$
\widehat{u}_{h}(t, x)=\int_{0}^{t}\left[\mathcal{S}_{h}(t-s) \widehat{W}(s, \cdot)\right](x) \mathrm{d} s \quad \text { a.s. }
$$

Proposition 5.1. Let $r \in\{1,2,3\}, \kappa(r) \in\{1, \ldots, r\}, w$ be the solution of (1.3) and $w_{h} \in S_{h}^{r, \kappa(r)}$ be its spacediscrete approximation given in (5.2). If $w_{0} \in \dot{\mathbf{H}}^{2}(D)$, then, there exists a constant $C>0$, independent of $T$ 
and $h$, such that

$$
\left(\int_{0}^{T}\left\|w-w_{h}\right\|_{0, D}^{2} \mathrm{~d} t\right)^{\frac{1}{2}} \leq C h^{\widetilde{\nu}(r, \theta)}\left\|w_{0}\right\|_{\dot{\mathbf{H}} \tilde{\xi}(r, \theta)} \quad \forall \theta \in[0,1]
$$

where:

(i) If $Q_{h}=\left(\Delta_{h}\right)^{2}$ and $\kappa(r) \geq 1$, then

$$
\widetilde{\nu}(r, \theta):=\left\{\begin{array}{ll}
(r+1) \theta & \text { if } r=1,2 \\
3 \theta & \text { if } r=3
\end{array} \quad \text { and } \quad \widetilde{\xi}(r, \theta):= \begin{cases}3 \theta-1 & \text { if } r=1,2 \\
2 \theta-1 & \text { if } r=3 .\end{cases}\right.
$$

(ii) If $Q_{h}=\left(\Delta_{h}\right)^{2}, r=3, \kappa(3) \geq 2$ and (2.21) holds, then

$$
\widetilde{\nu}(3, \theta):=4 \theta-1 \quad \text { and } \quad \widetilde{\xi}(3, \theta):=3 \theta-2 .
$$

(iii) If $Q_{h}=B_{h}$ and $\kappa(r) \geq 2$, then

$$
\widetilde{\nu}(r, \theta):=\left\{\begin{array}{ll}
2 \theta & \text { if } r=2 \\
4 \theta & \text { if } r=3
\end{array} \quad \text { and } \quad \widetilde{\xi}(r, \theta):= \begin{cases}3 \theta-2 & \text { if } r=2 \\
4 \theta-2 & \text { if } r=3 .\end{cases}\right.
$$

Proof. Let $e:=w-w_{h}$ and $\rho:=-\left(T_{B, h}-T_{B}\right) \partial_{x}^{4} w$, where $T_{B, h}=T_{B, h}^{I}$ or $T_{B, h}^{I I}$. We will derive (5.4) by interpolation, after showing that it holds for $\theta=1$ and $\theta=0$.

First, we can easily verify the error equation $T_{B, h} e_{t}+e=\rho$ on $[0, T]$. Then, using that $T_{B, h}$ is selfadjoint (see (2.26), (2.30)), $\left(T_{B, h} f, f\right)_{0, D} \geq 0$ for $f \in \mathrm{L}^{2}(D)$ and $T_{B, h} e(0)=0$, and proceeding as in Proposition 4.1, we obtain

$$
\int_{0}^{T}\|e\|_{0, D}^{2} \mathrm{~d} t \leq \int_{0}^{T}\|\rho\|_{0, D}^{2} \mathrm{~d} t
$$

Case 1. $Q_{h}=\Delta_{h}^{2}$

Let $r \in\{1,2\}$. Using (5.8), (2.28), (2.3) and (2.18) (with $\beta=\ell=0$ and $p=4$ ), we have

$$
\begin{aligned}
\int_{0}^{T}\|e\|_{0, D}^{2} \mathrm{~d} t & \leq C h^{2(r+1)} \int_{0}^{T}\|w\|_{4, D}^{2} \mathrm{~d} t \\
& \leq C h^{2(r+1)} \int_{0}^{T}\|w\|_{\dot{\mathbf{H}}^{4}}^{2} \mathrm{~d} t \\
& \leq C h^{2(r+1)}\left\|w_{0}\right\|_{\dot{\mathbf{H}}^{2}}^{2} .
\end{aligned}
$$

When $r=3$, then (5.8), (2.28), (2.3) and (2.18) (with $\beta=\ell=0$ and $p=3$ ) yield

$$
\begin{aligned}
\int_{0}^{T}\|e\|_{0, D}^{2} \mathrm{~d} t & \leq C h^{6} \int_{0}^{T}\|w\|_{3, D}^{2} \mathrm{~d} t \\
& \leq C h^{6} \int_{0}^{T}\|w\|_{\dot{\mathbf{H}}^{3}}^{2} \mathrm{~d} t \\
& \leq C h^{6}\left\|w_{0}\right\|_{\mathbf{H}^{1}}^{2}
\end{aligned}
$$

The relations (5.9) and (5.10) yield (5.4) with $\widetilde{\nu}$ and $\widetilde{\xi}$ given by (5.5) and (5.6) for $\theta=1$. Since $T_{B} w_{t}+w=0$ on $[0, T]$, we obtain $\left(T_{B} w_{t}, w\right)_{0, D}+\|w\|_{0, D}^{2}=0$ on $[0, T]$, which, along with $(2.16)$, yields $\frac{\mathrm{d}}{\mathrm{d} t}\left\|T_{E} w\right\|_{0, D}^{2}+$ $2\|w\|_{0, D}^{2}=0$ on $[0, T]$. Then, integrating over $[0, T]$ and using (2.12), we get

$$
\int_{0}^{T}\|w\|_{0, D}^{2} \mathrm{~d} t \leq C\left\|w_{0}\right\|_{-2, D}^{2}
$$


Since $Q_{h}^{-1}=\left.T_{B, h}^{I}\right|_{S_{h}^{r, \kappa(r)}}$, we obtain $T_{B, h}^{I} \partial_{t} w_{h}+w_{h}=0$ on $[0, T]$, and thus $\left(T_{B, h}^{I} \partial_{t} w_{h}, w_{h}\right)_{0, D}+\left\|w_{h}\right\|_{0, D}^{2}=0$ on $[0, T]$, which, along with (2.26), yields $\frac{\mathrm{d}}{\mathrm{d} t}\left\|T_{E, h} w_{h}\right\|_{0, D}^{2}+2\left\|w_{h}\right\|_{0, D}^{2}=0$ on $[0, T]$. Then, integrating over $[0, T]$ and using (2.1) and (2.25), we have

$$
\begin{aligned}
\int_{0}^{T}\left\|w_{h}\right\|_{0, D}^{2} \mathrm{~d} t & \leq\left\|T_{E, h} P_{h} w_{0}\right\|_{0, D}^{2} \\
& \leq C\left\|\left(T_{E, h} w_{0}\right)^{\prime}\right\|_{0, D}^{2} \\
& \leq C\left\|w_{0}\right\|_{-1, D}^{2} .
\end{aligned}
$$

Hence, from (5.11), (5.12) and (2.4), we obtain $\int_{0}^{T}\|e\|_{0, D}^{2} \mathrm{~d} t \leq C\left\|w_{0}\right\|_{\mathrm{H}^{-1}}^{2}$, which yields (5.4) and (5.5) with $\theta=0$. Let $r=3$ and $\kappa(3) \geq 2$. Then, when (2.21) holds, we have

$$
\begin{aligned}
\left\|\left(T_{E, h} w_{0}\right)^{\prime}\right\|_{0, D}^{2} & =\left(w_{0}, T_{E, h} w_{0}\right)_{0, D} \\
& \leq\left\|w_{0}\right\|_{-2, D}\left\|T_{E, h} w_{0}\right\|_{2, D} \\
& \leq C\left\|w_{0}\right\|_{-2, D} h^{-1}\left\|T_{E, h} w_{0}\right\|_{1, D} \\
& \leq C\left\|w_{0}\right\|_{-2, D} h^{-1}\left\|\left(T_{E, h} w_{0}\right)^{\prime}\right\|_{0, D}
\end{aligned}
$$

which, along with (2.1), yields

$$
\left\|\left(T_{E, h} w_{0}\right)^{\prime}\right\|_{0, D} \leq C h^{-1}\left\|w_{0}\right\|_{-2, D} .
$$

Now, we combine (5.11), (5.12), (5.13) and (2.4), to get $\int_{0}^{T}\|e\|_{0, D}^{2} \mathrm{~d} t \leq C h^{-1}\left\|w_{0}\right\|_{\mathrm{H}^{-2}}^{2}$, which yields (5.4) and (5.6) with $\theta=0$.

Case 2. $Q_{h}=B_{h}$

For $r=2$, using (5.8), (2.32), (2.3) and (2.18) (with $\beta=\ell=0$ and $p=3$ ), we have

$$
\begin{aligned}
\int_{0}^{T}\|e\|_{0, D}^{2} \mathrm{~d} t & \leq C h^{4} \int_{0}^{T}\|w\|_{3, D}^{2} \mathrm{~d} t \\
& \leq C h^{4} \int_{0}^{T}\|w\|_{\dot{\mathrm{H}}^{3}}^{2} \mathrm{~d} t \\
& \leq C h^{4}\left\|w_{0}\right\|_{\mathbf{H}^{1}}^{2} .
\end{aligned}
$$

Also, for $r=3$, using (5.8), (2.32), (2.3) and (2.18) (with $\beta=\ell=0$ and $p=4$ ), we have

$$
\begin{aligned}
\int_{0}^{T}\|e\|_{0, D}^{2} \mathrm{~d} t & \leq C h^{8} \int_{0}^{T}\|w\|_{4, D}^{2} \mathrm{~d} t \\
& \leq C h^{8} \int_{0}^{T}\|w\|_{\dot{\mathbf{H}}^{4}}^{2} \mathrm{~d} t \\
& \leq C h^{8}\left\|w_{0}\right\|_{\dot{\mathbf{H}}^{2}}^{2} .
\end{aligned}
$$

Hence, we got (5.4) and (5.7) for $\theta=1$.

Observing that $Q_{h}^{-1}=\left.T_{B, h}^{I I}\right|_{S_{h}^{r, \kappa(r)}}$, we obtain $T_{B, h}^{I I} \partial_{t} w_{h}+w_{h}=0$ on $[0, T]$, and thus we have $\left(T_{B, h}^{I I} \partial_{t} w_{h}, w_{h}\right)_{0, D}+$ $\left\|w_{h}\right\|_{0, D}^{2}=0$ on $[0, T]$. The latter equation, along with (2.30), yields $\frac{1}{2} \frac{\mathrm{d}}{\mathrm{d} t}\left(T_{B, h}^{I I} w_{h}, w_{h}\right)_{0, D}+\left\|w_{h}\right\|_{0, D}^{2}=0$ on $[0, T]$. 
Then, integrating over $[0, T]$ and using (2.31), we have

$$
\begin{aligned}
\int_{0}^{T}\left\|w_{h}\right\|_{0, D}^{2} \mathrm{~d} t & \leq \frac{1}{2}\left\|\left(T_{B, h}^{I I} P_{h} w_{0}\right)^{\prime \prime}\right\|_{0, D}^{2} \\
& \leq \frac{1}{2}\left\|\left(T_{B, h}^{I I} w_{0}\right)^{\prime \prime}\right\|_{0, D}^{2} \\
& \leq C\left\|w_{0}\right\|_{-2, D}^{2} .
\end{aligned}
$$

Hence, from (5.11), (5.16) and (2.4), we obtain $\int_{0}^{T}\|e\|_{0, D}^{2} \mathrm{~d} t \leq C\left\|w_{0}\right\|_{\mathbf{H}^{-2}}^{2}$, which yields (5.4) and (5.7) with $\theta=0$.

Next lemma shows that a discrete analogue of (1.4) holds.

Lemma 5.2. Let $r \in\{1,2,3\}, \kappa(r) \in\{1, \ldots, r\}$ and $w_{h}:[0, T] \rightarrow S_{h}^{r, \kappa(r)}$ be the space-discrete approximation of the solution $w$ of (1.3) defined in (5.2). Then, there exists a map $G_{h}:[0, T] \rightarrow C(\overline{D \times D})$ such that

$$
w_{h}(t ; x)=\int_{D} G_{h}(t ; x, y) w_{0}(y) \mathrm{d} y \quad \forall t \in[0, T], \quad \forall x \in \bar{D},
$$

and $G_{h}(t ; x, y)=G_{h}(t ; y, x)$ for $x, y \in \bar{D}$ and $t \in[0, T]$.

Proof. Let $\operatorname{dim}\left(S_{h}^{r, \kappa(r)}\right)=N_{h}$ and $\gamma_{h}: S_{h}^{r, \kappa(r)} \times S_{h}^{r, \kappa(r)} \rightarrow \mathbb{R}$ be an inner product on $S_{h}^{r, \kappa(r)}$ given by $\gamma_{h}\left(\chi_{A}, \chi_{B}\right):=$ $\left(\Delta_{h} \chi_{A}, \Delta_{h} \chi_{B}\right)_{0, D} \forall \chi_{A}, \chi_{B} \in S_{h}^{r, \kappa(r)}$ when $Q_{h}=\Delta_{h}^{2}$, and $\gamma_{h}\left(\chi_{A}, \chi_{B}\right):=\left(\chi_{A}^{\prime \prime}, \chi_{B}^{\prime \prime}\right)_{0, D} \forall \chi_{A}, \chi_{B} \in S_{h}^{r, \kappa(r)}$ when $Q_{h}=B_{h}$. We can construct a basis $\left(\chi_{j}\right)_{j=1}^{N_{h}}$ of $S_{h}^{r, \kappa(r)}$ which is $\mathrm{L}^{2}(D)$-orthonormal, i.e., $\left(\chi_{i}, \chi_{j}\right)_{0, D}=\delta_{i j}$ for $i, j=1, \ldots, N_{h}$, and $\gamma_{h}$-orthogonal, i.e., there are $\left(\lambda_{h, \ell}\right)_{\ell=1}^{N_{h}} \subset(0,+\infty)$ such that $\gamma_{h}\left(\chi_{i}, \chi_{j}\right)=\lambda_{h, i} \delta_{i j}$ for $i, j=1, \ldots, N_{h}$ (see Sect. 8.7 in [13]). Thus, there exists a map $\omega:[0, T] \rightarrow \mathbb{R}^{N_{h}}$ such that $w_{h}(t ; x)=$ $\sum_{j=1}^{N_{h}} \omega_{j}(t) \chi_{j}(x)$. Since $w_{h}(0)=P_{h} w_{0}$, it follows that $\omega_{j}(0)=\left(w_{0}, \chi_{j}\right)_{0, D}$ for $j=1, \ldots, N_{h}$. Now, (5.2) yields that $\frac{\mathrm{d}}{\mathrm{d} t} \omega(t)=B \omega(t)$ for $t \in[0, T]$, where $B \in \mathbb{R}^{N_{h} \times N_{h}}$ with $B_{i j}:=-\left(Q_{h} \chi_{j}, \chi_{i}\right)_{0, D}=-\gamma_{h}\left(\chi_{i}, \chi_{j}\right)=-\lambda_{h, i} \delta_{i j}$ for $i, j=1, \ldots, N_{h}$. Hence, it follows that

$$
\omega_{\ell}(t)=\mathrm{e}^{-\lambda_{h, \ell} t}\left(w_{0}, \chi_{\ell}\right)_{0, D} \quad \forall t \in[0, T], \quad \ell=1, \ldots, N_{h},
$$

which yields (5.17) with $G_{h}(t ; x, y)=\sum_{j=1}^{N_{h}} \mathrm{e}^{-\lambda_{h, j} t} \chi_{j}(x) \chi_{j}(y)$.

We are ready to derive a convergence estimate, in an $\mathrm{L}_{t}^{\infty}\left(\mathrm{L}_{P}^{2}\left(\mathrm{~L}_{x}^{2}\right)\right)$ norm, for the space-discrete approximation $\widehat{u}_{h}$ to the solution $\widehat{u}$ of the regularized problem.

Theorem 5.3. Let $r \in\{1,2,3\}, \kappa(r) \in\{1, \ldots, r\}$, $\widehat{u}$ be the solution of (1.6) and $\widehat{u}_{h}$ be its space-discrete approximation defined in (5.1). Then, there exist a constant $C>0$, independent of $T, \Delta t, \Delta x$ and $h$, such that

$$
\max _{[0, T]}\left(\mathbb{E}\left[\left\|\widehat{u}_{h}-\widehat{u}\right\|_{0, D}^{2}\right]\right)^{\frac{1}{2}} \leq C \epsilon^{-\frac{1}{2}} h^{\nu-\epsilon}, \quad \forall \epsilon \in(0, \nu],
$$

where:

(i) If $Q_{h}=\left(\Delta_{h}\right)^{2}$ and $\kappa(r) \geq 1$, then

$$
\nu:= \begin{cases}\frac{r+1}{6} & \text { if } r=1,2 \\ \frac{3}{4} & \text { if } r=3 .\end{cases}
$$

(ii) If $Q_{h}=\left(\Delta_{h}\right)^{2}, r=3, \kappa(3) \geq 2$ and (2.21) holds, then

$$
\nu:=1 .
$$


(iii) If $Q_{h}=B_{h}$ and $\kappa(r) \geq 2$, then

$$
\nu:= \begin{cases}1 & \text { if } r=2 \\ \frac{3}{2} & \text { if } r=3\end{cases}
$$

Proof. Let $\widehat{e}:=\widehat{u}_{h}-\widehat{u}$ and $t \in(0, T]$. Then, (5.3), (5.17) and (1.8) yield

$$
\widehat{e}(t, x)=\int_{0}^{T} \int_{D} \mathcal{X}_{(0, t)}(s)\left[G_{h}(t-s ; x, y)-G(t-s ; x, y)\right] \widehat{W}(s, y) \mathrm{d} s \mathrm{~d} y \quad \forall x \in \bar{D}, \quad \text { a.s. }
$$

Thus, using Lemma 2.1, the Itô isometry property of the stochastic integral and (2.9), we obtain

$$
\mathbb{E}\left[\|e(t, \cdot)\|_{0, D}^{2}\right] \leq \int_{0}^{t}\left(\int_{D} \int_{D}\left[G_{h}(t-s ; x, y)-G(t-s ; x, y)\right]^{2} \mathrm{~d} y \mathrm{~d} x\right) \mathrm{d} s
$$

which, along with (2.5), yields

$$
\mathbb{E}\left[\|e(t, \cdot)\|_{0, D}^{2}\right] \leq \int_{0}^{t}\left\|\mathcal{S}(s)-\mathcal{S}_{h}(s)\right\|_{\mathrm{HS}}^{2} \mathrm{~d} s .
$$

Since $e(0, \cdot)=0$, we use $(5.22)$, the definition of the Hilbert-Schmidt norm and (5.4), to obtain

$$
\begin{aligned}
\max _{[0, T]} \mathbb{E}\left[\|e\|_{0, D}^{2}\right] & \leq \int_{0}^{T}\left(\sum_{k=1}^{\infty}\left\|\mathcal{S}(s) \varepsilon_{k}-\mathcal{S}_{h}(s) \varepsilon_{k}\right\|_{0, D}^{2}\right) \mathrm{d} s \\
& \leq \sum_{k=1}^{\infty}\left(\int_{0}^{T}\left\|\mathcal{S}(s) \varepsilon_{k}-\mathcal{S}_{h}(s) \varepsilon_{k}\right\|_{0, D}^{2} \mathrm{~d} s\right) \\
& \leq C h^{2 \widetilde{\nu}(r, \theta)} \sum_{k=1}^{\infty}\left\|\varepsilon_{k}\right\|_{\dot{\mathbf{H}} \tilde{\xi}(r, \theta)}^{2} \\
& \leq C h^{2 \widetilde{\nu}(r, \theta)} \sum_{k=1}^{\infty} \lambda_{k}^{2 \widetilde{\xi}(r, \theta)}
\end{aligned}
$$

To finish the proof we have to find the values of $\theta \in[0,1]$ which ensure the convergence of the series in the right hand side of (5.23), which is equivalent to the requirement $-2 \widetilde{\xi}(r, \theta)>1$. Thus, using (2.7), we arrive at the bound (5.18) with value $\nu$ given by (5.19), (5.20) and (5.21).

\section{Convergence of the fully-Discrete approximations}

\subsection{Consistency estimates}

First, we derive some Hölder-type bounds for $\widehat{u}$.

Lemma 6.1. Let $\widehat{u}$ be the solution of (1.6). Then, there exist a real positive constant $C$, which is independent of $T, \Delta t$ and $\Delta x$, such that

$$
\left\{\mathbb{E}\left[\left\|\int_{\tau_{a}}^{\tau_{b}}\left[\widehat{u}\left(\tau_{b}, \cdot\right)-\widehat{u}(\tau, \cdot)\right] \mathrm{d} \tau\right\|_{0, D}^{2}\right]\right\}^{\frac{1}{2}} \leq C\left[1+\left(\tau_{b}-\tau_{a}\right)^{\frac{1}{4}}\right]^{\frac{1}{2}}\left|\tau_{b}-\tau_{a}\right|^{1+\frac{3}{8}}
$$

and

$$
\left\{\mathbb{E}\left[\left\|\widehat{u}\left(\tau_{b}, \cdot\right)-\widehat{u}\left(\tau_{a}, \cdot\right)\right\|_{0, D}^{2}\right]\right\}^{\frac{1}{2}} \leq C\left[1+\left(\tau_{b}-\tau_{a}\right)^{\frac{1}{4}}\right]^{\frac{1}{2}}\left|\tau_{b}-\tau_{a}\right|^{\frac{3}{8}}
$$

for $\tau_{a}, \tau_{b} \in[0, T]$ with $\tau_{a} \leq \tau_{b}$. 
Proof. We will omit the proof of (6.2) because it is similar to that of (6.1) which follows.

Let $m \in\{1, \ldots, M\}, \tau_{b} \in(0, T]$ and $\tau_{a} \in[0, T]$ with $\tau_{a}<\tau_{b}$, and $\mu(\cdot):=\int_{\tau_{a}}^{\tau_{b}}\left[\widehat{u}\left(\tau_{b}, \cdot\right)-\widehat{u}(\tau, \cdot)\right] \mathrm{d} \tau$. First we assume that $\tau_{a}>0$. Then, we use (1.8), Lemma 2.1, the Itô-isometry property of the stochastic integral, (1.5) and the $\mathrm{L}^{2}(D)$-orthogonality of $\left(\varepsilon_{k}\right)_{k=1}^{\infty}$, to obtain

$$
\begin{aligned}
\mathbb{E}\left[\|\mu\|_{0, D}^{2}\right] & \leq \frac{1}{\Delta t} \sum_{n=1}^{N_{\Delta}} \int_{D} \int_{D}\left[\int_{T_{n}} \int_{\tau_{a}}^{\tau_{b}}\left[\mathcal{X}_{\left(0, \tau_{b}\right)}\left(s^{\prime}\right) G\left(\tau_{b}-s^{\prime} ; x, y\right)-\mathcal{X}_{(0, \tau)}\left(s^{\prime}\right) G\left(\tau-s^{\prime} ; x, y\right)\right] \mathrm{d} \tau \mathrm{d} s^{\prime}\right]^{2} \mathrm{~d} x \mathrm{~d} y \\
& \leq \frac{1}{\Delta t} \sum_{n=1}^{N_{\Delta}} \sum_{k=1}^{\infty}\left(\int_{T_{n}} \int_{\tau_{a}}^{\tau_{b}}\left[\mathcal{X}_{\left(0, \tau_{b}\right)}\left(s^{\prime}\right) \mathrm{e}^{-\lambda_{k}^{4}\left(\tau_{b}-s^{\prime}\right)}-\mathcal{X}_{(0, \tau)}\left(s^{\prime}\right) \mathrm{e}^{-\lambda_{k}^{4}\left(\tau-s^{\prime}\right)}\right] \mathrm{d} \tau \mathrm{d} s^{\prime}\right)^{2}
\end{aligned}
$$

which, along with the use of the Cauchy-Schwarz inequality, yields

$$
\begin{aligned}
\mathbb{E}\left[\|\mu\|_{0, D}^{2}\right] & \leq \sum_{k=1}^{\infty} \int_{0}^{T}\left[\int_{\tau_{a}}^{\tau_{b}}\left[\mathcal{X}_{\left(0, \tau_{b}\right)}\left(s^{\prime}\right) \mathrm{e}^{-\lambda_{k}^{4}\left(\tau_{b}-s^{\prime}\right)}-\mathcal{X}_{(0, \tau)}\left(s^{\prime}\right) \mathrm{e}^{-\lambda_{k}^{4}\left(\tau-s^{\prime}\right)}\right] \mathrm{d} \tau\right]^{2} \mathrm{~d} s^{\prime} \\
& \leq\left(\tau_{b}-\tau_{a}\right) \sum_{k=1}^{\infty}\left(\int_{\tau_{a}}^{\tau_{b}} \int_{0}^{\tau}\left[\mathrm{e}^{-\lambda_{k}^{4}\left(\tau_{b}-s^{\prime}\right)}-\mathrm{e}^{-\lambda_{k}^{4}\left(\tau-s^{\prime}\right)}\right]^{2} \mathrm{~d} s^{\prime} \mathrm{d} \tau+\int_{\tau_{a}}^{\tau_{b}} \int_{\tau}^{\tau_{b}} \mathrm{e}^{-2 \lambda_{k}^{4}\left(\tau_{b}-s^{\prime}\right)} \mathrm{d} s^{\prime} \mathrm{d} \tau\right) \\
& \leq\left(\tau_{b}-\tau_{a}\right)^{2} \sum_{k=1}^{\infty} \frac{1-\mathrm{e}^{-2 \lambda_{k}^{4}\left(\tau_{b}-\tau_{a}\right)}}{\lambda_{k}^{4}} .
\end{aligned}
$$

Finally, we combine (3.13) and (6.3) to arrive at (6.1). The case $\tau_{a}=0$ follows by moving along the lines of the proof above using that $\widehat{u}(0, x)=0$.

Next, we show a consistency result for the Backward Euler time-discrete approximations of $\widehat{u}$, which is based on the result of Lemma 6.1.

Proposition 6.2. Let $\widehat{u}$ be the solution of (1.6) and $\left(\widehat{\sigma}_{m}\right)_{m=1}^{M}$ be stochastic functions defined by

$$
\widehat{u}\left(\tau_{m}, \cdot\right)-\widehat{u}\left(\tau_{m-1}, \cdot\right)+k_{m} \partial_{x}^{4} \widehat{u}\left(\tau_{m}, \cdot\right)=\int_{\Delta_{m}} \widehat{W} \mathrm{~d} \tau+\widehat{\sigma}_{m} \quad \text { a.s. }, \quad m=1, \ldots, M .
$$

Then it holds that

$$
\left\{\mathbb{E}\left[\left\|T_{B} \widehat{\sigma}_{m}\right\|_{0, D}^{2}\right]\right\}^{\frac{1}{2}} \leq C\left(1+k_{m}^{\frac{1}{4}}\right)^{\frac{1}{2}}\left(k_{m}\right)^{1+\frac{3}{8}}, \quad m=1, \ldots, M,
$$

where $C$ is the constant in Lemma 6.1.

Proof. Let $m \in\{1, \ldots, M\}$. Integrating the equation in (1.6) over $\Delta_{m}$ and subtracting it from (6.4), we conclude that $T_{B} \widehat{\sigma}_{m}(\cdot)=\int_{\Delta_{m}}\left[\widehat{u}\left(\tau_{m}, \cdot\right)-\widehat{u}(\tau, \cdot)\right] \mathrm{d} \tau$ a.s. Thus, to get the bound (6.5), we apply the result (6.1) on the latter equality.

\subsection{Discrete in time $\mathbf{L}_{t}^{2}\left(\mathbf{L}_{P}^{2}\left(\mathbf{L}_{x}^{2}\right)\right)$ error estimate}

We first obtain a discrete in time $\mathrm{L}_{t}^{2}\left(\mathrm{~L}_{P}^{2}\left(\mathrm{~L}_{x}^{2}\right)\right)$ error estimate for the Backward Euler fully-discrete approximations of $\widehat{u}$, by connecting it to the error estimate of Theorem 5.3 for the space-discrete approximation of $\widehat{u}$.

Theorem 6.3. Let $r \in\{1,2,3\}, \kappa(r) \in\{1, \ldots, r\}$, $\widehat{u}$ be the solution of (1.6) and $\left(\widehat{U}_{h}^{m}\right)_{m=0}^{M} \subset S_{h}^{r, \kappa(r)}$ be the Backward Euler fully-discrete approximations of $\widehat{u}$ defined in (1.12)-(1.13). Then there exists a constant $C>0$, 
independent of $T, \Delta t, \Delta x, h, M$ and $\left(k_{m}\right)_{m=1}^{M}$, such that:

$$
\left\{\sum_{m=1}^{M} k_{m} \mathbb{E}\left[\left\|\widehat{U}_{h}^{m}-\widehat{u}\left(\tau_{m}, \cdot\right)\right\|_{0, D}^{2}\right]\right\}^{\frac{1}{2}} \leq C \sqrt{T}\left[\epsilon^{-\frac{1}{2}} h^{\nu-\epsilon}+\widehat{\omega}\left(k_{\max }\right)\left(k_{\max }\right)^{\frac{3}{8}}\right], \quad \forall \epsilon \in(0, \nu],
$$

where $\widehat{\omega}\left(k_{\max }\right):=\left(1+\left(k_{\max }\right)^{\frac{1}{4}}\right)^{\frac{1}{2}}$ and $\nu$ is defined in Theorem 5.3.

Proof. Let $\widehat{u}_{h}$ be the space-discrete approximation of $\widehat{u}$ defined in (5.1), $\widehat{e}=\widehat{u}-\widehat{u}_{h}, z_{h}^{m}:=\widehat{U}_{h}^{m}-\widehat{u}_{h}\left(\tau_{m}\right) \in S_{h}^{r, \kappa(r)}$ for $m=0, \ldots, M$, and $V_{h}:=\left\{\sum_{m=1}^{M} k_{m} \mathbb{E}\left[\left\|z_{h}^{m}\right\|_{0, D}^{2}\right]\right\}^{\frac{1}{2}}$. First, we observe that

$$
\left\{\sum_{m=1}^{M} k_{m} \mathbb{E}\left[\left\|\widehat{U}_{h}^{m}-\widehat{u}\left(\tau_{m}, \cdot\right)\right\|_{0, D}^{2}\right]\right\}^{\frac{1}{2}} \leq V_{h}+\sqrt{T} \max _{[0, T]}\left\{\mathbb{E}\left[\|\widehat{e}\|_{0, D}^{2}\right]\right\}^{\frac{1}{2}}
$$

Integrating (5.1) over $\Delta_{m}$ and subtracting the obtained relation from (1.13), we arrive at

$$
T_{B, h}\left(z_{h}^{m}-z_{h}^{m-1}\right)+k_{m} z_{h}^{m}=\rho_{h, m} \quad \text { a.s., } \quad m=1, \ldots, M,
$$

where $\rho_{h, m}:=\int_{\Delta_{m}}\left[\widehat{u}_{h}(\tau, \cdot)-\widehat{u}_{h}\left(\tau_{m}, \cdot\right)\right] \mathrm{d} \tau, T_{B, h}=T_{B, h}^{I}$ when $Q_{h}=\Delta_{h}^{2}$, and $T_{B, h}=T_{B, h}^{I I}$ when $Q_{h}=B_{h}$. Take the $(\cdot, \cdot)_{0, D}$-inner product of both sides of $(6.8)$ with $z_{h}^{m}$, sum with respect to $m$ from 1 up to $M$, and use (2.26) to obtain

$$
\sum_{m=1}^{M}\left(T_{B, h}\left(z_{h}^{m}-z_{h}^{m-1}\right), z_{h}^{m}\right)_{0, D}+\sum_{m=1}^{M} k_{m}\left\|z_{h}^{m}\right\|_{0, D}^{2}=\sum_{m=1}^{M}\left(\rho_{h, m}, z_{h}^{m}\right)_{0, D} \quad \text { a.s. }
$$

Since $z_{h}^{0}=0$, we conclude that

$$
\begin{aligned}
\sum_{m=1}^{M}\left(T_{B, h}^{I}\left(z_{h}^{m}-z_{h}^{m-1}\right), z_{h}^{m}\right)_{0, D} & =\sum_{m=1}^{M}\left(T_{E, h} z_{h}^{m}-T_{E, h} z_{h}^{m-1}, T_{E, h} z_{h}^{m}\right)_{0, D} \\
& \geq \frac{1}{2}\left\|T_{E, h} z_{h}^{M}\right\|_{0, D}^{2} \quad \text { a.s. }
\end{aligned}
$$

when $Q_{h}=\Delta_{h}^{2}$, and that

$$
\begin{aligned}
\sum_{m=1}^{M}\left(T_{B, h}^{I I}\left(z_{h}^{m}-z_{h}^{m-1}\right), z_{h}^{m}\right)_{0, D} & =\sum_{m=1}^{M}\left(\left(T_{B, h}^{I I} z_{h}^{m}\right)^{\prime \prime}-\left(T_{B, h}^{I I} z_{h}^{m-1}\right)^{\prime \prime},\left(T_{B, h}^{I I} z_{h}^{m}\right)^{\prime \prime}\right)_{0, D} \\
& \geq \frac{1}{2}\left\|\left(T_{B, h}^{I I} z_{h}^{M}\right)^{\prime \prime}\right\|_{0, D}^{2} \quad \text { a.s., }
\end{aligned}
$$

when $Q_{h}=B_{h}$. Thus, taking expected values in (6.9), using (6.10) or (6.11), and applying the Cauchy-Schwarz inequality we get

$$
\begin{aligned}
\left(V_{h}\right)^{2} & \leq \mathbb{E}\left[\sum_{m=1}^{M} k_{m}^{-1}\left\|\rho_{h, m}\right\|_{0, D}^{2}\right] \\
& \leq \mathbb{E}\left[\sum_{m=1}^{M} \int_{D} \int_{\Delta_{m}}\left[\widehat{u}_{h}(\tau, x)-\widehat{u}_{h}\left(\tau_{m}, x\right)\right]^{2} \mathrm{~d} \tau \mathrm{d} x\right] \\
& \leq \sum_{m=1}^{M} \int_{\Delta_{m}} \mathbb{E}\left[\left\|\widehat{u}_{h}(\tau, \cdot)-\widehat{u}_{h}\left(\tau_{m}, \cdot\right)\right\|_{0, D}^{2}\right] \mathrm{d} \tau .
\end{aligned}
$$


Using (6.12) and (6.2), we conclude that

$$
\begin{aligned}
V_{h} & \leq\left\{\sum_{m=1}^{M} \int_{\Delta_{m}} \mathbb{E}\left[\left\|\widehat{e}(\tau, \cdot)-\widehat{e}\left(\tau_{m}, \cdot\right)\right\|_{0, D}^{2}\right] \mathrm{d} \tau\right\}^{\frac{1}{2}}+\left\{\sum_{m=1}^{M} \int_{\Delta_{m}} \mathbb{E}\left[\left\|\widehat{u}(\tau, \cdot)-\widehat{u}\left(\tau_{m}, \cdot\right)\right\|_{0, D}^{2}\right] \mathrm{d} \tau\right\}^{\frac{1}{2}} \\
& \leq 2 \sqrt{T} \max _{[0, T]}\left\{\mathbb{E}\left[\|\widehat{e}\|_{0, D}^{2}\right]\right\}^{\frac{1}{2}}+C \sqrt{T} \widehat{\omega}\left(k_{\max }\right)\left(k_{\max }\right)^{\frac{3}{8}} .
\end{aligned}
$$

Thus, (6.6) follows from (6.7), (6.13) and (5.18).

In the sequel, we will derive an alternative discrete in time $\mathrm{L}_{t}^{2}\left(\mathrm{~L}_{P}^{2}\left(\mathrm{~L}_{x}^{2}\right)\right)$ a priori error estimate for the Backward Euler fully-discrete approximations of $\widehat{u}$. Before that, let us state a useful lemma for the proof of which we refer the reader to Appendix C.

Lemma 6.4. Let $\widehat{u}$ be the solution to the problem (1.6). If there exists a constant $c_{0}>0$ such that

$$
\min _{1 \leq n \leq N_{\Delta}} \Delta t_{n} \geq c_{0} \Delta t
$$

then there exists a constant $C>0$, independent of $T, \Delta t$ and $\Delta x$, such that

$$
\max _{[0, T]}\left\{\mathbb{E}\left[\left\|\partial_{x}^{3} \widehat{u}\right\|_{0, D}^{2}\right]\right\}^{\frac{1}{2}} \leq C\left[1+(\Delta t)^{-\frac{3}{8}}\right] .
$$

Theorem 6.5. Let $r \in\{1,2,3\}, \kappa(r) \in\{1, \ldots, r\}$, $\widehat{u}$ be the solution of (1.6) and $\left(\widehat{U}_{h}^{m}\right)_{m=0}^{M} \subset S_{h}^{r, \kappa(r)}$ be the Backward Euler fully-discrete approximations of $\widehat{u}$ defined in (1.12)-(1.13). If there exists a constant $c_{0}>0$ such that $\min _{1 \leq n \leq N_{\Delta}} \Delta t_{n} \geq c_{0} \Delta t$, then there exists a constant $C>0$, independent of $T, \Delta t, \Delta x, h, M$ and $\left(k_{m}\right)_{m=1}^{M}$, such that

$$
\left\{\sum_{m=1}^{M} k_{m} \mathbb{E}\left[\left\|\widehat{u}\left(\tau_{m}, \cdot\right)-\widehat{U}_{h}^{m}\right\|_{0, D}^{2}\right]\right\}^{\frac{1}{2}} \leq C \sqrt{T}\left[h^{r}+h^{r}(\Delta t)^{-\frac{3}{8}}+\widehat{\omega}\left(k_{\max }\right)\left(k_{\max }\right)^{\frac{3}{8}}\right],
$$

where $\widehat{\omega}\left(k_{\max }\right):=\left(1+\left(k_{\max }\right)^{\frac{1}{4}}\right)^{\frac{1}{2}}$.

Proof. First, set $\zeta^{m}:=\widehat{u}\left(\tau_{m}, \cdot\right)-\widehat{U}_{h}^{m}$ for $m=0, \ldots, M$. Then, subtract (1.13) from (6.4) to get

$$
T_{B, h}\left(\zeta^{m}-\zeta^{m-1}\right)+k_{m} \zeta^{m}=\xi_{1, m}+\xi_{2, m} \quad \text { a.s., } \quad m=1, \ldots, M,
$$

where $\xi_{1, m}:=-\left(T_{B, h}-T_{B}\right)\left(\int_{\Delta_{m}} \partial_{x}^{4} \widehat{u} \mathrm{~d} s\right), \xi_{2, m}:=T_{B} \widehat{\sigma}^{m}, T_{B, h}=T_{B, h}^{I}$ when $Q_{h}=\Delta_{h}^{2}$, and $T_{B, h}=T_{B, h}^{I I}$ when $Q_{h}=B_{h}$. Proceeding as in the proof of Theorem 6.3 we arrive at

$$
\left\{\sum_{m=1}^{M} k_{m} \mathbb{E}\left[\left\|\zeta^{m}\right\|_{0, D}^{2}\right]\right\}^{\frac{1}{2}} \leq \widetilde{V}_{1}+\widetilde{V}_{2}
$$

where $\widetilde{V}_{1}:=\left\{\sum_{m=1}^{M} k_{m}^{-1} \mathbb{E}\left[\left\|\xi_{1, m}\right\|_{0, D}^{2}\right]\right\}^{\frac{1}{2}}$ and $\widetilde{V}_{2}:=\left\{\sum_{m=1}^{M} k_{m}^{-1} \mathbb{E}\left[\left\|\xi_{2, m}\right\|_{0, D}^{2}\right]\right\}^{\frac{1}{2}}$. Applying (6.5), we obtain

$$
\widetilde{V}_{2} \leq C \sqrt{T} \widehat{\omega}\left(k_{\max }\right)\left(k_{\max }\right)^{\frac{3}{8}}
$$


Using the Cauchy-Schwarz inequality and the estimates (2.28) and (2.32), we have

$$
\begin{aligned}
\widetilde{V}_{1} & \leq C h^{r}\left\{\sum_{m=1}^{M} k_{m}^{-1} \mathbb{E}\left[\left\|\int_{\Delta_{m}} \partial_{x}^{4} \widehat{u} \mathrm{~d} \tau\right\|_{-1, D}^{2}\right]\right\}^{\frac{1}{2}} \\
& \leq C h^{r}\left\{\sum_{m=1}^{M} k_{m}^{-1} \mathbb{E}\left[\left(\int_{\Delta_{m}}\left\|\partial_{x}^{4} \widehat{u}\right\|_{-1, D} \mathrm{~d} \tau\right)^{2}\right]\right\}^{\frac{1}{2}} \\
& \leq C h^{r}\left\{\sum_{m=1}^{M} \mathbb{E}\left[\int_{\Delta_{m}}\left\|\partial_{x}^{3} \widehat{u}\right\|_{0, D}^{2} \mathrm{~d} \tau\right]\right\}^{\frac{1}{2}} \\
& \leq C h^{r} \sqrt{T} \max _{[0, T]}\left\{\mathbb{E}\left[\left\|\partial_{x}^{3} \widehat{u}\right\|_{0, D}^{2}\right]\right\}^{\frac{1}{2}} .
\end{aligned}
$$

Thus, (6.15) follows from (6.17), (6.18), (6.19) and (6.14).

\subsection{Discrete in time $\mathbf{L}_{t}^{\infty}\left(\mathbf{L}_{P}^{2}\left(\mathbf{L}_{x}^{2}\right)\right)$ error estimate}

To get a discrete in time $\mathrm{L}_{t}^{\infty}\left(\mathrm{L}_{P}^{2}\left(\mathrm{~L}_{x}^{2}\right)\right)$ error estimate for the Backward Euler fully-discrete approximations of $\widehat{u}$, we compare them to the Backward Euler time-discrete approximations of $\widehat{u}$ defined in (4.1)-(4.2). This is obtained via a discrete in time $\mathrm{L}_{t}^{2}\left(\mathrm{~L}_{x}^{2}\right)$ comparison estimate between: (i) the Backward Euler time-discrete approximations of the solution $w$ of (1.3) defined in (4.3)-(4.4), and (ii) the Backward Euler fully-discrete approximations of the solution $w$ of (1.3) specified below:

Step DFD1. Set

$$
W_{h}^{0}:=P_{h} w_{0}
$$

Step DFD2. For $m=1, \ldots, M$, find $W_{h}^{m} \in S_{h}^{r, \kappa(r)}$ such that

$$
W_{h}^{m}-W_{h}^{m-1}+k_{m} Q_{h} W_{h}^{m}=0
$$

Proposition 6.6. Let $r \in\{1,2,3\}, \kappa(r) \in\{1, \ldots, r\}, w$ be the solution of the problem $(1.3)$, $\left(W^{m}\right)_{m=0}^{M}$ be the Backward Euler time-discrete approximations of $w$ defined in $(4.3)-(4.4)$, and $\left(W_{h}^{m}\right)_{m=0}^{M}$ be the Backward Euler fully-discrete approximations of $w$ specified in (6.20)-(6.21). If $w_{0} \in \dot{\mathbf{H}}^{2}(D)$, then, there exists a constant $C>0$, independent of $T, h, M$ and $\left(k_{m}\right)_{m=1}^{M}$, such that

$$
\left(\sum_{m=1}^{M} k_{m}\left\|W^{m}-W_{h}^{m}\right\|_{0, D}^{2}\right)^{\frac{1}{2}} \leq C h^{\widetilde{\nu}(r, \theta)}\left\|w_{0}\right\|_{\dot{\mathbf{H}} \tilde{\xi}(r, \theta)} \quad \forall \theta \in[0,1],
$$

where $\widetilde{\nu}(r, \theta)$ and $\widetilde{\xi}(r, \theta)$ are defined in Proposition 5.1 .

Proof. The bound (6.22) follows by interpolation, showing it first for $\theta=0$ and $\theta=1$.

Let $E^{m}:=W^{m}-W_{h}^{m}$ for $m=0, \ldots, M$. We use (4.4) and (6.21), to obtain: $T_{B, h}\left(E^{m}-E^{m-1}\right)+k_{m} E^{m}=$ $k_{m}\left(T_{B}-T_{B, h}\right) \partial_{x}^{4} W^{m}$ for $m=1, \ldots, M$, where: $T_{B, h}=T_{B, h}^{I}$ when $Q_{h}=\Delta_{h}^{2}$, and $T_{B, h}=T_{B, h}^{I I}$ when $Q_{h}=B_{h}$. Since $T_{E, h} E^{0}=0$ and $T_{B, h}^{I I} E^{0}=0$, proceeding as in the proof of Theorem 6.3 , it follows that

$$
\sum_{m=1}^{M} k_{m}\left\|E^{m}\right\|_{0, D}^{2} \leq \sum_{m=1}^{M} k_{m}\left\|\left(T_{B}-T_{B, h}\right) \partial_{x}^{4} W^{m}\right\|_{0, D}^{2}
$$


Case 1. $Q_{h}=\Delta_{h}^{2}$

Let $r=1$ or 2 . Then, by (2.28) and (6.23), we obtain

$$
\sum_{m=1}^{M} k_{m}\left\|E^{m}\right\|_{0, D}^{2} \leq C h^{2(r+1)} \sum_{m=1}^{M} k_{m}\left\|\partial_{x}^{4} W^{m}\right\|_{0, D}^{2}
$$

Taking the $(\cdot, \cdot)_{0, D}$-inner product of $(4.4)$ with $\partial_{x}^{4} W^{m}$, and then integrating by parts and summing with respect to $m$ from 1 up to $M$, it follows that

$$
\sum_{m=1}^{M}\left(\partial_{x}^{2} W^{m}-\partial_{x}^{2} W^{m-1}, \partial_{x}^{2} W^{m}\right)_{0, D}+\sum_{m=1}^{M} k_{m}\left\|\partial_{x}^{4} W^{m}\right\|_{0, D}^{2}=0
$$

Since $\sum_{m=1}^{M}\left(\partial_{x}^{2} W^{m}-\partial_{x}^{2} W^{m-1}, \partial_{x}^{2} W^{m}\right)_{0, D} \geq \frac{1}{2}\left[\left\|\partial_{x}^{2} W^{M}\right\|_{0, D}^{2}-\left\|\partial_{x}^{2} W^{0}\right\|_{0, D}^{2}\right],(6.25)$ yields

$$
\sum_{m=1}^{M} k_{m}\left\|\partial_{x}^{4} W^{m}\right\|_{0, D}^{2} \leq \frac{1}{2}\left\|w_{0}\right\|_{2, D}^{2}
$$

Combining, now, (6.24), (6.26) and (2.3), we obtain

$$
\left(\sum_{m=1}^{M} k_{m}\left\|E^{m}\right\|_{0, D}^{2}\right)^{\frac{1}{2}} \leq C h^{r+1}\left\|w_{0}\right\|_{\dot{\mathrm{H}}^{2}}
$$

Let $r=3$. Then, by $(2.28)$ and (6.23), we obtain

$$
\sum_{m=1}^{M} k_{m}\left\|E^{m}\right\|_{0, D}^{2} \leq C h^{6} \sum_{m=1}^{M} k_{m}\left\|\partial_{x}^{3} W^{m}\right\|_{0, D}^{2}
$$

Taking the $(\cdot, \cdot)_{0, D}$-inner product of $(4.4)$ with $\partial_{x}^{2} W^{m}$, and then integrating by parts and summing with respect to $m$ from 1 up to $M$, it follows that

$$
\sum_{m=1}^{M}\left(\partial_{x} W^{m}-\partial_{x} W^{m-1}, \partial_{x} W^{m}\right)_{0, D}+\sum_{m=1}^{M} k_{m}\left\|\partial_{x}^{3} W^{m}\right\|_{0, D}^{2}=0
$$

Since $\sum_{m=1}^{M}\left(\partial_{x} W^{m}-\partial_{x} W^{m-1}, \partial_{x} W^{m}\right)_{0, D} \geq \frac{1}{2}\left[\left\|\partial_{x} W^{M}\right\|_{0, D}^{2}-\left\|\partial_{x} W^{0}\right\|_{0, D}^{2}\right],(6.29)$ yields

$$
\sum_{m=1}^{M} k_{m}\left\|\partial_{x}^{3} W^{m}\right\|_{0, D}^{2} \leq \frac{1}{2}\left\|w_{0}\right\|_{1, D}^{2}
$$

Combining (6.28), (6.30) and (2.3) we get

$$
\left(\sum_{m=1}^{M} k_{m}\left\|E^{m}\right\|_{0, D}^{2}\right)^{\frac{1}{2}} \leq C h^{3}\left\|w_{0}\right\|_{\dot{\mathbf{H}}^{1}} .
$$

Thus, the relations (6.27) and (6.31) yield (6.22) and (5.5) for $\theta=1$. 
Since $T_{B, h}\left(W_{h}^{m}-W_{h}^{m-1}\right)+k_{m} W_{h}^{m}=0$ for $m=1, \ldots, M$, we obtain $\left(T_{B, h}\left(W_{h}^{m}-W_{h}^{m-1}\right), W_{h}^{m}\right)_{0, D}+$ $k_{m}\left\|W_{h}^{m}\right\|_{0, D}^{2}$ for $m=1, \ldots, M$, which, along with (2.26), yields

$$
\sum_{m=1}^{M} k_{m}\left\|W_{h}^{m}\right\|_{0, D}^{2} \leq \frac{1}{2}\left\|T_{E, h} w_{0}\right\|_{0, D}^{2}
$$

Now, using (2.25), (2.4) and (6.32), we obtain

$$
\sum_{m=1}^{M} k_{m}\left\|W_{h}^{m}\right\|_{0, D}^{2} \leq C\left\|w_{0}\right\|_{\dot{\mathbf{H}}^{-1}}^{2}
$$

Finally, combine (6.33) with (4.10) to get $\left(\sum_{m=1}^{M} k_{m}\left\|E^{m}\right\|_{0, D}^{2}\right)^{\frac{1}{2}} \leq C\left\|w_{0}\right\|_{\dot{\mathrm{H}}^{-1}}$, which is equivalent to (6.22) and (5.5) for $\theta=0$. Let $r=3$ and $\kappa(3) \geq 2$. Then, when (2.21) holds, we combine (6.32), (2.1), (5.13) and (2.4) to get

$$
\sum_{m=1}^{M} k_{m}\left\|W_{h}^{m}\right\|_{0, D}^{2} \leq C h^{-2}\left\|w_{0}\right\|_{\dot{\mathbf{H}}^{-2}}^{2} .
$$

Thus, (6.34) with (4.10) give $\left(\sum_{m=1}^{M} k_{m}\left\|E^{m}\right\|_{0, D}^{2}\right)^{\frac{1}{2}} \leq C h^{-1}\left\|w_{0}\right\|_{\dot{\mathbf{H}}^{-2}}$, which is equivalent to (6.22) and (5.6) for $\theta=0$.

Case 2. $Q_{h}=B_{h}$

For $r=2$, using (2.32), (6.23), (6.30) and (2.3), we have

$$
\left(\sum_{m=1}^{M} k_{m}\left\|E^{m}\right\|_{0, D}^{2}\right)^{\frac{1}{2}} \leq C h^{2}\left\|w_{0}\right\|_{\dot{\mathbf{H}}^{1}}
$$

Also, for $r=3$, using (2.32), (6.23), (6.26) and (2.3), it follows that

$$
\left(\sum_{m=1}^{M} k_{m}\left\|E^{m}\right\|_{0, D}^{2}\right)^{\frac{1}{2}} \leq C h^{4}\left\|w_{0}\right\|_{\dot{\mathbf{H}}^{2}} .
$$

Thus, (6.35) and (6.36) yields (6.22) and (5.7) for $\theta=1$.

Since $T_{B, h}^{I I}\left(W_{h}^{m}-W_{h}^{m-1}\right)+k_{m} W_{h}^{m}=0$ for $m=1, \ldots, M$, we conclude that $\left(T_{B, h}^{I I}\left(W_{h}^{m}-W_{h}^{m-1}\right), W_{h}^{m}\right)_{0, D}+$ $k_{m}\left\|W_{h}^{m}\right\|_{0, D}^{2}=0$ for $m=1, \ldots, M$, which yields

$$
\left(\left(T_{B, h}^{I I} W_{h}^{m}\right)^{\prime \prime}-\left(T_{B, h}^{I I} W_{h}^{m-1}\right)^{\prime \prime},\left(T_{B, h}^{I I} W_{h}^{m}\right)^{\prime \prime}\right)_{0, D}+k_{m}\left\|W_{h}^{m}\right\|_{0, D}^{2}=0, \quad m=1, \ldots, M .
$$

Then, sum with respect to $m$, and use (2.31) and (2.4), to obtain

$$
\begin{aligned}
\sum_{m=1}^{M} k_{m}\left\|W_{h}^{m}\right\|_{0, D}^{2} & \leq \frac{1}{2}\left\|\left(T_{B, h} w_{0}\right)^{\prime \prime}\right\|_{0, D}^{2} \\
& \leq C\left\|w_{0}\right\|_{-2, D}^{2} \\
& \leq C\left\|w_{0}\right\|_{\dot{\mathbf{H}}^{-2}}^{2} .
\end{aligned}
$$

Finally, combine (6.37) with (4.10) to get $\left(\sum_{m=1}^{M} k_{m}\left\|E^{m}\right\|_{0, D}^{2}\right)^{\frac{1}{2}} \leq C\left\|w_{0}\right\|_{\dot{H}^{-2}}$, which yields (6.22) and (5.7) for $\theta=0$. 
The following lemma ensures the existence of a continuous Green function for the solution operator of a discrete elliptic problem.

Lemma 6.7. Let $r \in\{1,2,3\}, \kappa(r) \in\{1, \ldots, r\}, \epsilon>0, f \in \mathrm{L}^{2}(D)$ and $\psi_{h} \in S_{h}^{r, \kappa(r)}$ such that

$$
\epsilon Q_{h} \psi_{h}+\psi_{h}=P_{h} f
$$

where $Q_{h}=\Delta_{h}^{2}$ when $k(r) \geq 1$, or $Q_{h}=B_{h}$ when $\kappa(r) \geq 2$. Then there exists a function $G_{h, \epsilon} \in C(\overline{D \times D})$ such that

$$
\psi_{h}(x)=\int_{D} G_{h, \epsilon}(x, y) f(y) \mathrm{d} y \quad \forall x \in \bar{D}
$$

and $G_{h, \epsilon}(x, y)=G_{h, \epsilon}(y, x)$ for $x, y \in \bar{D}$.

Proof. Keeping the notation and the constructions of the proof of Lemma 5.2, we conclude that there are $\left(\mu_{j}\right)_{j=1}^{N_{h}} \subset \mathbb{R}$ such that $\psi_{h}=\sum_{j=1}^{N_{h}} \mu_{j} \chi_{j}$. Thus, (6.38) is equivalent to $\mu_{i}=\frac{1}{1+\epsilon \lambda_{h, i}}\left(f, \chi_{i}\right)_{0, D}$ for $i=1, \ldots, N_{h}$. Finally, we obtain (6.39) with $G_{h, \epsilon}(x, y)=\sum_{j=1}^{N_{h}} \frac{\chi_{j}(x) \chi_{j}(y)}{1+\epsilon \lambda_{h, j}}$.

We are ready to compare, in the discrete in time $\mathrm{L}_{t}^{\infty}\left(\mathrm{L}_{P}^{2}\left(\mathrm{~L}_{x}^{2}\right)\right)$ norm, the time-discrete with the fully-discrete Backward Euler approximations of $\widehat{u}$.

Proposition 6.8. Let $r \in\{1,2,3\}, \kappa(r) \in\{1, \ldots, r\}, \widehat{u}$ be the solution of the problem (1.6), $\left(\widehat{U}_{h}^{m}\right)_{m=0}^{M} \subset$ $S_{h}^{r, \kappa(r)}$ be the Backward Euler fully-discrete approximations of $\widehat{u}$ specified in (1.12)-(1.13), and $\left(\widehat{U}^{m}\right)_{m=0}^{M}$ be the Backward Euler time-discrete approximations of $\widehat{u}$ specified in (4.1)-(4.2). If the partition $\left(\tau_{m}\right)_{m=0}^{M}$ is uniform, i.e. $k_{m}=\Delta \tau$ for $m=1, \ldots, M$, then there exists a constant $C>0$, independent of $\Delta x, \Delta t, h, M$ and $\Delta \tau$, such that

$$
\max _{1 \leq m \leq M}\left\{\mathbb{E}\left[\left\|\widehat{U}_{h}^{m}-\widehat{U}^{m}\right\|_{0, D}^{2}\right]\right\}^{\frac{1}{2}} \leq C \epsilon^{-\frac{1}{2}} h^{\nu-\epsilon}, \quad \forall \epsilon \in(0, \nu],
$$

where $\nu$ has been specified in Theorem 5.3.

Proof. Let $I: \mathrm{L}^{2}(D) \rightarrow \mathrm{L}^{2}(D)$ be the identity operator and $\Lambda_{h}: \mathrm{L}^{2}(D) \rightarrow S_{h}^{r, \kappa(r)}$ be the inverse discrete elliptic operator given by $\Lambda_{h}:=\left(I+\Delta \tau Q_{h}\right)^{-1} P_{h}$ and having a Green function $G_{h, \Delta \tau}$ (cf. Lem. 6.7). Also, for $\ell \in \mathbb{N}$, we denote by $G_{h, \Delta, \ell}$ the Green function of $\Lambda_{h}^{\ell}$. Using, now, an induction argument, from (1.13) we conclude that $\widehat{U}_{h}^{m}=\sum_{j=1}^{m} \int_{\Delta_{j}} \Lambda_{h}^{m-j+1} \widehat{W}(\tau, \cdot) \mathrm{d} \tau, m=1, \ldots, M$, which is written, equivalently, as follows:

$$
\widehat{U}_{h}^{m}(x)=\int_{0}^{\tau_{m}} \int_{D} \widehat{\mathcal{D}}_{h, m}(\tau ; x, y) \widehat{W}(\tau, y) \mathrm{d} y \mathrm{~d} \tau \quad \forall x \in \bar{D}, \quad m=1, \ldots, M,
$$

where

$$
\widehat{\mathcal{D}}_{h, m}(\tau ; x, y):=\sum_{j=1}^{m} \mathcal{X}_{\Delta_{j}}(\tau) G_{h, \Delta \tau, m-j+1}(x, y) \quad \forall \tau \in[0, T], \quad \forall x, y \in D .
$$

Using (4.13), (6.41), Lemma 2.1, the Itô-isometry property of the stochastic integral, (2.5) and (2.9), we get

$$
\begin{aligned}
\mathbb{E}\left[\left\|\widehat{U}^{m}-\widehat{U}_{h}^{m}\right\|_{0, D}^{2}\right] & \leq \int_{0}^{\tau_{m}}\left(\int_{D} \int_{D}\left[\widehat{\mathcal{K}}_{m}(\tau ; x, y)-\widehat{\mathcal{D}}_{h, m}(\tau ; x, y)\right]^{2} \mathrm{~d} y \mathrm{~d} x\right) \mathrm{d} \tau \\
& \leq \sum_{j=1}^{m} \int_{\Delta_{j}}\left\|\Lambda^{m-j+1}-\Lambda_{h}^{m-j+1}\right\|_{\mathrm{HS}}^{2} \mathrm{~d} \tau, \quad m=1, \ldots, M,
\end{aligned}
$$


where $\Lambda$ is the inverse elliptic operator defined in the proof of Theorem 4.2. Now, we use the definition of the Hilbert-Schmidt norm and the deterministic error estimate (6.22), to have

$$
\begin{aligned}
\mathbb{E}\left[\left\|\widehat{U}^{m}-\widehat{U}_{h}^{m}\right\|_{0, D}^{2}\right] & \leq \sum_{j=1}^{m} \Delta \tau\left[\sum_{k=1}^{\infty}\left\|\Lambda^{m-j+1} \varepsilon_{k}-\Lambda_{h}^{m-j+1} \varepsilon_{k}\right\|_{0, D}^{2}\right] \\
& \leq \sum_{k=1}^{\infty}\left[\sum_{j=1}^{m} \Delta \tau\left\|\Lambda^{j} \varepsilon_{k}-\Lambda_{h}^{j} \varepsilon_{k}\right\|_{0, D}^{2}\right] \\
& \leq C h^{2 \widetilde{\nu}(r) \theta} \sum_{k=1}^{\infty}\left\|\varepsilon_{k}\right\|_{\dot{\mathbf{H}} \tilde{\xi}(r, \theta)}^{2}, \quad m=1, \ldots, M, \quad \forall \theta \in[0,1] .
\end{aligned}
$$

Thus, we arrive at

$$
\max _{1 \leq m \leq M} \mathbb{E}\left[\left\|\widehat{U}^{m}-\widehat{U}_{h}^{m}\right\|_{0, D}^{2}\right] \leq C h^{2 \widetilde{\nu}(r) \theta} \sum_{k=1}^{\infty} \lambda_{k}^{2 \widetilde{\xi}(r, \theta)}, \quad \forall \theta \in[0,1],
$$

from which, requiring $-2 \widetilde{\xi}(r, \theta)>1$, (6.40), easily, follows ( $c f$. Thm. 5.3).

The available error estimates allow us to conclude a discrete in time $\mathrm{L}_{t}^{\infty}\left(\mathrm{L}_{P}^{2}\left(\mathrm{~L}_{x}^{2}\right)\right)$ convergence of the Backward Euler fully-discrete approximations of $\widehat{u}$, over a uniform partition of $[0, T]$.

Theorem 6.9. Let $r \in\{1,2,3\}, \kappa(r) \in\{1, \ldots, r\}, \nu$ be defined in Theorem 5.3, $\widehat{u}$ be the solution of problem (1.6), and $\left(\widehat{U}_{h}^{m}\right)_{m=0}^{M} \subset S_{h}^{r, \kappa(r)}$ be the Backward Euler fully-discrete approximations of $\widehat{u}$ constructed by (1.12)(1.13). If the partition $\left(\tau_{m}\right)_{m=0}^{M}$ is uniform, i.e., $k_{m}=\Delta \tau$ for $m=1, \ldots, M$, then there exists a constant $C>0$, independent of $T, h, \Delta \tau, \Delta t$ and $\Delta x$, such that

$$
\max _{0 \leq m \leq M}\left\{\mathbb{E}\left[\left\|\widehat{U}_{h}^{m}-\widehat{u}\left(\tau_{m}, \cdot\right)\right\|_{0, D}^{2}\right]\right\}^{\frac{1}{2}} \leq C\left[\widetilde{\omega}\left(\Delta \tau, \epsilon_{1}\right) \epsilon_{1}^{-\frac{1}{2}}(\Delta \tau)^{\frac{3}{8}-\epsilon_{1}}+\epsilon_{2}^{-\frac{1}{2}} h^{\nu-\epsilon_{2}}\right]
$$

for $\epsilon_{1} \in\left(0, \frac{3}{8}\right]$ and $\epsilon_{2} \in(0, \nu]$, where $\widetilde{\omega}\left(\Delta \tau, \epsilon_{1}\right):=1+(\Delta \tau)^{\epsilon_{1}}\left(1+(\Delta \tau)^{\frac{1}{4}}\right)^{\frac{1}{2}}$.

Proof. The estimate is a simple consequence of the error bounds (6.40) and (4.12).

Below, adopting a viewpoint similar to that in Theorem 6.5 , we are able we show an alternative discrete in time $\mathrm{L}_{t}^{\infty}\left(\mathrm{L}_{P}^{2}\left(\mathrm{~L}_{x}^{2}\right)\right)$ error estimate.

Theorem 6.10. Let $r \in\{2,3\}, \kappa(r) \in\{1, \ldots, r\}, \widehat{u}$ be the solution of problem (1.6), and $\left(\widehat{U}_{h}^{m}\right)_{m=0}^{M} \subset S_{h}^{r, \kappa(r)}$ be the Backward Euler fully-discrete approximations of $\widehat{u}$ constructed by $(1.12)-(1.13)$. If the partition $\left(\tau_{m}\right)_{m=0}^{M}$ is uniform, i.e., $k_{m}=\Delta \tau$ for $m=1, \ldots, M$, then there exists a constant $C>0$, independent of $T, h, \Delta \tau, \Delta t$ and $\Delta x$, such that

$$
\max _{0 \leq m \leq M}\left\{\mathbb{E}\left[\left\|\widehat{U}_{h}^{m}-\widehat{u}\left(\tau_{m}, \cdot\right)\right\|_{0, D}^{2}\right]\right\}^{\frac{1}{2}} \leq C\left[\widetilde{\omega}(\Delta \tau, \epsilon) \epsilon^{-\frac{1}{2}}(\Delta \tau)^{\frac{3}{8}-\epsilon}+h^{r} \Delta \tau^{-1}\right]
$$

for $\epsilon \in\left(0, \frac{3}{8}\right]$, where $\widetilde{\omega}(\Delta \tau, \epsilon):=1+(\Delta \tau)^{\epsilon}\left(1+(\Delta \tau)^{\frac{1}{4}}\right)^{\frac{1}{2}}$.

Proof. Set $\widehat{Z}^{m}:=\widehat{U}^{m}-\widehat{U}_{h}^{m}$ for $m=0, \ldots, M$, and combine (4.2) and (1.13) to get the error equation: $T_{B, h}\left(Z^{m}-Z^{m-1}\right)+\Delta \tau Z^{m}=\Delta \tau\left(T_{B}-T_{B, h}\right) \partial_{x}^{4} \widehat{U}^{m}$ for $m=1, \ldots, M$, where: $T_{B, h}=T_{B, h}^{I}$ when $Q_{h}=\Delta_{h}^{2}$, and $T_{B, h}=T_{B, h}^{I I}$ when $Q_{h}=B_{h}$. Proceed now as in the proof of Proposition 6.6 to arrive at $\sum_{m=1}^{M} \Delta \tau \mathbb{E}\left[\left\|Z^{m}\right\|_{0, D}^{2}\right] \leq$ $C \sum_{m=1}^{M} \Delta \tau \mathbb{E}\left[\left\|\left(T_{B}-T_{B, h}\right) \partial_{x}^{4} \widehat{U}^{m}\right\|_{0, D}^{2}\right]$, which after using (2.28) and (2.32) yields

$$
\max _{0 \leq m \leq M}\left(\mathbb{E}\left[\left\|Z^{m}\right\|_{0, D}^{2}\right]\right)^{\frac{1}{2}} \leq C h^{r} \Delta \tau^{-\frac{1}{2}} \max _{1 \leq m \leq M}\left(\mathbb{E}\left[\left\|\partial_{x}^{3} \widehat{U}^{m}\right\|_{0, D}^{2}\right]\right)^{\frac{1}{2}} .
$$


Since it holds that (see Appendix D)

$$
\max _{1 \leq m \leq M}\left(\mathbb{E}\left[\left\|\partial_{x}^{3} \widehat{U}^{m}\right\|_{0, D}^{2}\right]\right)^{\frac{1}{2}} \leq C \Delta \tau^{-\frac{1}{2}}
$$

(6.43) yields $\max _{0 \leq m \leq M}\left(\mathbb{E}\left[\left\|Z^{m}\right\|_{0, D}^{2}\right]\right)^{\frac{1}{2}} \leq C h^{r} \Delta \tau^{-\frac{1}{2}}$ which along with (4.12) establish (6.42).

Remark 6.11. Let us assume that $h=O(\Delta \tau)$. Then the order of convergence with respect to $h$ in (6.42) becomes equal to $r-1$, which is greater than $\nu$ for $r \in\{2,3\}$ (cf. Thm. 5.3).

\section{A. Appendix A}

Proof of Proposition 2.2. Let $f \in \mathrm{L}^{2}(D)$. Then, we consider the following cases with respect to $r$.

Case 1. $r \in\{1,2\}$

Using (2.15) and (2.24) we split the error as follows

$$
\left\|T_{B} f-T_{B, h}^{I} f\right\|_{0, D} \leq\left\|E_{1}\right\|_{0, D}+\left\|E_{2}\right\|_{0, D},
$$

where $E_{1}:=\left(T_{E}-T_{E, h}\right) T_{E} f$ and $E_{2}:=T_{E, h}\left(T_{E} f-T_{E, h} f\right)$. First, we use (2.27) and (2.12) to obtain

$$
\begin{aligned}
\left\|E_{1}\right\|_{0, D} & \leq C h^{r+1}\left\|T_{E}\left(T_{E} f\right)\right\|_{r+1, D} \\
& \leq C h^{r+1}\left\|T_{E} f\right\|_{r-1, D} \\
& \leq C h^{r+1}\|f\|_{r-3, D} .
\end{aligned}
$$

To handle $\left\|E_{2}\right\|_{0, D}$, we apply (2.1) and (2.25) to get

$$
\begin{aligned}
\left\|E_{2}\right\|_{0, D} & \leq C\left\|\left(E_{2}\right)^{\prime}\right\|_{0, D} \\
& \leq C\left\|E_{\star}\right\|_{-1, D},
\end{aligned}
$$

with $E_{\star}:=T_{E} f-T_{E, h} f \in \mathrm{H}_{0}^{1}(D)$. Let $\varphi \in \dot{\mathbf{H}}^{1}(D)=\mathrm{H}_{0}^{1}(D)$ and $w=T_{E} \varphi$. Then, using the Galerkin orthogonality along with (2.19), (2.27), (2.1), (2.25) and (2.12), we obtain

$$
\begin{aligned}
\left(\varphi, E_{\star}\right)_{0, D} & =-\left(w^{\prime}, E_{\star}^{\prime}\right)_{0, D} \\
& \leq\left\|E_{\star}\right\|_{1, D} \inf _{\chi \in S_{h}^{r, \kappa(r)}}\|w-\chi\|_{1, D} \\
& \leq C h^{s-1}\left\|T_{E} f\right\|_{s, D} h^{r}\left\|T_{E} \varphi\right\|_{r+1, D} \\
& \leq C h^{r+s-1}\|f\|_{s-2, D}\|\varphi\|_{r-1, D}, \quad s=1,2,
\end{aligned}
$$

from which, we conclude that

$$
\left\|E_{\star}\right\|_{-1, D} \leq C h^{r+\ell+1}\|f\|_{\ell, D}, \quad \ell=-1,0 .
$$

Thus, the estimate (2.28) for $r \in\{1,2\}$ is a simple consequence of (A.1), (A.2), (A.3) and (A.4).

Case 2. $r=3$

We split the error in the following way

$$
\left\|T_{B} f-T_{B, h}^{I} f\right\|_{0, D} \leq\left\|\mathcal{E}_{1}\right\|_{0, D}+\left\|\mathcal{E}_{2}\right\|_{0, D}
$$


where $\mathcal{E}_{1}:=\left(T_{E}-T_{E, h}\right) T_{E, h} f$ and $\mathcal{E}_{2}:=T_{E}\left(T_{E} f-T_{E, h} f\right)$. Using (2.27), (2.12), (2.1) and (2.25), we conclude that

$$
\begin{aligned}
\left\|\mathcal{E}_{1}\right\|_{0, D} & \leq C h^{3}\left\|T_{E} T_{E, h} f\right\|_{3, D} \\
& \leq C h^{3}\left\|T_{E, h} f\right\|_{1, D} \\
& \leq C h^{3}\|f\|_{-1, D} .
\end{aligned}
$$

Now, we use (2.12) to obtain

$$
\left\|\mathcal{E}_{2}\right\|_{0, D} \leq C\left\|E_{\star}\right\|_{-2, D},
$$

where $E_{\star}:=T_{E} f-T_{E, h} f \in \mathrm{H}_{0}^{1}(D)$. Let $\varphi \in \dot{\mathbf{H}}^{2}(D)=\mathrm{H}_{0}^{1}(D) \cap \mathrm{H}^{2}(D)$ and $w=T_{E} \varphi$. Then, using (2.19), (2.27), (2.1), (2.25) and (2.12) we have

$$
\begin{aligned}
\left(\varphi, E_{\star}\right)_{0, D} & =-\left(w^{\prime}, E_{\star}^{\prime}\right)_{0, D} \\
& \leq\left\|E_{\star}\right\|_{1, D} \inf _{\chi \in S_{h}^{3, \kappa(3)}}\|w-\chi\|_{1, D} \\
& \leq C\left\|T_{E} f\right\|_{1, D} h^{3}\left\|T_{E} \varphi\right\|_{4, D} \\
& \leq C h^{3}\|f\|_{-1, D}\|\varphi\|_{2, D} .
\end{aligned}
$$

Thus, we conclude that

$$
\left\|E_{\star}\right\|_{-2, D} \leq C h^{3}\|f\|_{-1, D} .
$$

We combine, now, (A.5), (A.6), (A.7) and (A.8), to arrive at (2.28) for $r=3$.

\section{B. Appendix B}

Proof of Proposition 2.3. Let $r \in\{2,3\}, \kappa(r) \geq 2, f \in \mathrm{L}^{2}(D)$ and $e=T_{B} f-T_{B, h}^{I I} f$. Since, $\left(e^{\prime \prime}, \chi^{\prime \prime}\right)_{0, D}=0$ for all $\chi \in S_{h}^{r, \kappa(r)}$, using (2.20) and (2.14) we conclude that

$$
\begin{aligned}
\left\|e^{\prime \prime}\right\|_{0, D} & \leq C h^{s-1}\left\|T_{B} f\right\|_{s+1, D} \\
& \leq C h^{s-1}\|f\|_{s-3, D}, \quad s=2, \ldots, r .
\end{aligned}
$$

Case 1. $r=3$

Let $w \in \dot{\mathbf{H}}^{4}(D)$ be defined by $T_{B} e=w$. Then, using (B.1), (2.20) and (2.14), we have:

$$
\begin{aligned}
\|e\|_{0, D}^{2} & =\left(w^{\prime \prime}, e^{\prime \prime}\right)_{0, D} \\
& \leq\left\|e^{\prime \prime}\right\|_{0, D} \inf _{\chi \in S_{h}^{3, k(3)}}\|w-\chi\|_{2, D} \\
& \leq C h^{s-1}\|f\|_{s-3, D} h^{2}\|w\|_{4, D} \\
& \leq C h^{s+1}\|f\|_{s-3, D}\|e\|_{0, D}, \quad s=2,3,
\end{aligned}
$$

which yields (2.32) for $r=3$. 
Case 2. $r=2$

Let $w \in \dot{\mathbf{H}}^{2}(D)$ be defined by $T_{E} e=w$. Then, using (B.1), (2.20), (2.14), (2.12) and (2.1), we have:

$$
\begin{aligned}
\left\|e^{\prime}\right\|_{0, D}^{2} & =-\left(w^{\prime \prime}, e^{\prime \prime}\right)_{0, D} \\
& \leq\left\|e^{\prime \prime}\right\|_{0, D} \inf _{\chi \in S_{h}^{2, \kappa(2)}}\|w-\chi\|_{2, D} \\
& \leq C h^{2}\|f\|_{-1, D}\|w\|_{3, D} \\
& \leq C h^{2}\|f\|_{-1, D}\|e\|_{1, D},
\end{aligned}
$$

which yields

$$
\|e\|_{1, D} \leq C h^{2}\|f\|_{-1, D}
$$

To arrive at (2.32) for $r=2$, we combine (B.2) and (2.1).

\section{Appendix C}

Proof of Lemma 6.4. Let $\Theta_{\Delta}:=\left\{\mathbb{E}\left[\left\|\partial_{x}^{3} \widehat{u}\right\|_{0, D}^{2}\right]\right\}^{\frac{1}{2}}$ and $t \in(0, T]$. Also, we adopt some notation introduced in the proof of Theorem 3.1. Using (1.8), Lemma 2.1, the Itô isometry property of the stochastic integral and (2.9), we obtain

$$
\left(\Theta_{\Delta}\right)^{2}(t) \leq \sum_{n=1}^{\widehat{N}(t)} \frac{1}{\Delta t_{n}} \int_{D} \int_{D}\left(\int_{\widehat{T}_{n}(t)} \partial_{x}^{3} G\left(t-s^{\prime} ; x, y\right) \mathrm{d} s^{\prime}\right)^{2} \mathrm{~d} x \mathrm{~d} y .
$$

Let $\left(\varphi_{k}\right)_{k=1}^{\infty}$ be a sequence of functions defined by $\varphi_{k}(x):=\sqrt{2} \cos \left(\lambda_{k} x\right) \forall x \in \bar{D}$. Now, we use (1.5) and the $(\cdot, \cdot)_{0, D}$-orthonormality of $\left(\varphi_{k}\right)_{k=1}^{\infty}$ and $\left(\varepsilon_{k}\right)_{k=1}^{\infty}$ to have

$$
\begin{aligned}
\left(\Theta_{\Delta}\right)^{2}(t) & \leq \sum_{n=1}^{\widehat{N}(t)} \frac{1}{\Delta t_{n}} \int_{D} \int_{D}\left[\sum_{k=1}^{\infty} \lambda_{k}^{3} \varphi_{k}(x) \varepsilon_{k}(y) \int_{\widehat{T}_{n}(t)} \mathrm{e}^{-\lambda_{k}^{4}\left(t-s^{\prime}\right)} \mathrm{d} s^{\prime}\right]^{2} \mathrm{~d} x \mathrm{~d} y \\
& \leq \sum_{k=1}^{\infty} \lambda_{k}^{6}\left\{\sum_{n=1}^{\widehat{N}(t)} \frac{1}{\Delta t_{n}}\left(\int_{\widehat{T}_{n}(t)} \mathrm{e}^{-\lambda_{k}^{4}\left(t-s^{\prime}\right)} \mathrm{d} s^{\prime}\right)^{2}\right\} \\
& \leq \sum_{k=1}^{\infty} \frac{1}{\lambda_{k}^{2}}\left\{\sum_{n=1}^{\widehat{N}(t)-1} \frac{1}{\Delta t_{n}}\left[\mathrm{e}^{-\lambda_{k}^{4}\left(t-t_{n}\right)}-\mathrm{e}^{-\lambda_{k}^{4}\left(t-t_{n-1}\right)}\right]^{2}+\frac{1}{\Delta t_{\widehat{N}(t)}}\left[1-\mathrm{e}^{-\lambda_{k}^{4}\left(t-t_{\widehat{N}(t)-1}\right)}\right]^{2}\right\} \\
& \leq \sum_{k=1}^{\infty} \frac{1}{\lambda_{k}^{2}}\left\{\sum_{n=1}^{\widehat{N}(t)-1} \frac{1}{\Delta t_{n}} \mathrm{e}^{-2 \lambda_{k}^{4}\left(t-t_{n}\right)}\left[1-\mathrm{e}^{-\lambda_{k}^{4}\left(t_{n}-t_{n-1}\right)}\right]^{2}+\frac{1}{\Delta t_{\widehat{N}(t)}}\left[1-\mathrm{e}^{-\lambda_{k}^{4}\left(t-t_{\widehat{N}(t)-1}\right)}\right]^{2}\right\} .
\end{aligned}
$$

Hence, we arrive at

$$
\left(\Theta_{\Delta}\right)^{2}(t) \leq \sum_{k=1}^{\infty} \frac{\left(1-\mathrm{e}^{-\lambda_{k}^{4} \Delta t}\right)^{2}}{\lambda_{k}^{2}} \Upsilon_{k}(t)
$$

where

$$
\Upsilon_{k}(t):=\frac{1}{\Delta t_{\widehat{N}(t)}}+\sum_{n=1}^{\widehat{N}(t)-1} \frac{1}{\Delta t_{n}} \mathrm{e}^{-2 \lambda_{k}^{4}\left(t-t_{n}\right)} \quad \forall k \in \mathbb{N}
$$


For $k \in \mathbb{N}$, we bound $\Upsilon_{k}(t)$ as follows

$$
\begin{aligned}
\Upsilon_{k}(t) & \leq \frac{1}{\Delta t_{\widehat{N}(t)}}+\sum_{n=1}^{\widehat{N}(t)-2} \frac{1}{\Delta t_{n} \Delta t_{n+1}} \int_{t_{n}}^{t_{n+1}} \mathrm{e}^{-2 \lambda_{k}^{4}(t-s)} \mathrm{d} s+\frac{1}{\Delta t_{\widehat{N}(t)-1}} \mathrm{e}^{-2 \lambda_{k}^{4}\left(t-t_{\widehat{N}(t)-1}\right)} \\
& \leq C \frac{1}{\Delta t^{2}}\left[\Delta t+\int_{0}^{t} \mathrm{e}^{-2 \lambda_{k}^{4}(t-s)} \mathrm{d} s\right] \\
& \leq C \frac{1}{\Delta t^{2}}\left[\Delta t+\frac{1}{2 \lambda_{k}^{4}}\left(1-\mathrm{e}^{-2 \lambda_{k}^{4} t}\right)\right] .
\end{aligned}
$$

Combining (C.2) and (C.1) we arrive at

$$
\left(\Theta_{\Delta}\right)^{2}(t) \leq C\left[\frac{1}{\Delta t} \sum_{k=1}^{\infty} \frac{\left(1-\mathrm{e}^{-\lambda_{k}^{4} \Delta t}\right)^{2}}{\lambda_{k}^{2}}+\frac{1}{2(\Delta t)^{2}} \sum_{k=1}^{\infty} \frac{\left(1-\mathrm{e}^{-\lambda_{k}^{4} \Delta t}\right)^{2}}{\lambda_{k}^{6}}\right],
$$

which, along with the inequality inequality $1-\mathrm{e}^{-\lambda_{k}^{4} \Delta t} \leq \min \left\{\lambda_{k}^{4} \Delta t, 1\right\}$ and the fact $\Theta_{\Delta}(0)=0$, yields

$$
\max _{[0, T]}\left(\Theta_{\Delta}\right)^{2} \leq \frac{C}{\Delta t} \sum_{k=1}^{\infty} \frac{1-\mathrm{e}^{-\lambda_{k}^{4} \Delta t}}{\lambda_{k}^{2}}
$$

In order to find how the right hand side of (C.3) depends on $\Delta t$, we proceed as in the proof of Theorem 3.1 (cf. (3.13)) to obtain

$$
\sum_{k=1}^{\infty} \frac{1-\mathrm{e}^{-\lambda_{k}^{4} \Delta t}}{\lambda_{k}^{2}} \leq 2 \pi^{2} \Delta t+\frac{8}{\pi}(\Delta t)^{\frac{1}{4}}
$$

Thus, the bound (6.14) follows as a simple consequence of (C.3) and (C.4).

\section{Appendix D}

Proof of (6.44). Let $\Lambda$ be the inverse elliptic operator introduced in the proof of Theorem 4.2, $\delta=\Delta \tau$ and $m \in\{1, \ldots, N\}$. Moving along the lines of the proof of Proposition 6.8, we obtain

$$
\begin{aligned}
\mathbb{E}\left[\left\|\partial_{x}^{3} \widehat{U}^{m}\right\|_{0, D}^{2}\right] & \leq \sum_{k=1}^{\infty} \delta\left(\sum_{j=1}^{m}\left\|\partial_{x}^{3} \Lambda^{j} \varepsilon_{k}\right\|_{0, D}^{2}\right) \\
& \leq \sum_{k=1}^{\infty} \delta \lambda_{k}^{6}\left(\sum_{j=1}^{m} \frac{1}{\left(1+\delta \lambda_{k}^{4}\right)^{2 j}}\right) \\
& \leq \sum_{k=1}^{\infty} \delta \lambda_{k}^{6}\left[\frac{1-\frac{1}{\left(1+\delta \lambda_{k}^{4}\right)^{2 m}}}{\delta^{2} \lambda_{k}^{8}+2 \delta \lambda_{k}^{4}}\right] \\
& \leq \sum_{k=1}^{\infty} \frac{1}{\delta \lambda_{k}^{2}}\left[1-\frac{1}{\left(1+\delta \lambda_{k}^{4}\right)^{2 m}}\right]
\end{aligned}
$$

which, obviously, yields $\mathbb{E}\left[\left\|\partial_{x}^{3} \widehat{U}^{m}\right\|_{0, D}^{2}\right] \leq \frac{1}{\delta} \sum_{k=1}^{\infty} \frac{1}{\lambda_{k}^{2}}=O\left(\delta^{-1}\right)$. 


\section{REFERENCES}

[1] E.J. Allen, S.J. Novosel and Z. Zhang, Finite element and difference approximation of some linear stochastic partial differential equations. Stoch. Stoch. Rep. 64 (1998) 117-142.

[2] I. Babuška, R. Tempone and G.E. Zouraris, Galerkin finite element approximations of stochastic elliptic partial differential equations. SIAM J. Numer. Anal. 42 (2004) 800-825.

[3] L. Bin, Numerical method for a parabolic stochastic partial differential equation. Master Thesis 2004-03, Chalmers University of Technology, Göteborg, Sweden (2004).

[4] J.H. Bramble and S.R. Hilbert, Estimation of linear functionals on Sobolev spaces with application to Fourier transforms and spline interpolation. SIAM J. Numer. Anal. 7 (1970) 112-124.

[5] S.C. Brenner and L.R. Scott, The Mathematical Theory of Finite Element Methods. Springer-Verlag, New York, USA (1994).

[6] C. Cardon-Weber, Implicit approximation scheme for the Cahn-Hilliard stochastic equation. PMA 613, Laboratoire de Probabilités et Modèles Alétoires, CNRS U.M.R. 7599, Universtités Paris VI et VII, Paris, France (2000).

[7] C. Cardon-Weber, Cahn-Hilliard equation: existence of the solution and of its density. Bernoulli 7 (2001) 777-816.

[8] P.G. Ciarlet, The finite element methods for elliptic problems. North-Holland, New York (1987).

[9] H. Cook, Browian motion in spinodal decomposition. Acta Metall. 18 (1970) 297-306.

[10] G. Da Prato and A. Debussche, Stochastic Cahn-Hilliard equation. Nonlinear Anal. 26 (1996) 241-263.

[11] N. Dunford and J.T. Schwartz, Linear Operators. Part II. Spectral Theory. Self Adjoint Operators in Hilbert Space. Reprint of the 1963 original, Wiley Classics Library, A Wiley-Interscience Publication, John Wiley \& Sons, Inc., New York, USA (1988).

[12] K.R. Elder, T.M. Rogers and R.C. Desai, Numerical study of the late stages of spinodal decomposition. Phys. Rev. B $\mathbf{3 7}$ (1987) 9638-9649.

[13] G.H. Golub and C.F. Van Loan, Matrix Computations. Second Edition, The John Hopkins University Press, Baltimore, USA (1989).

[14] W. Grecksch and P.E. Kloeden, Time-discretised Galerkin approximations of parabolic stochastic PDEs. Bull. Austral. Math. Soc. 54 (1996) 79-85.

[15] E. Hausenblas, Numerical analysis of semilinear stochastic evolution equations in Banach spaces. J. Comput. Appl. Math. 147 (2002) 485-516.

[16] E. Hausenblas, Approximation for semilinear stochastic evolution equations. Potential Anal. 18 (2003) 141-186.

[17] G. Kallianpur and J. Xiong, Stochastic Differential Equations in Infinite Dimensional Spaces, Lecture Notes-Monograph Series 26. Institute of Mathematical Statistics, Hayward, USA (1995).

[18] L. Kielhorn and M. Muthukumar, Spinodal decomposition of symmetric diblock copolymer homopolymer blends at the Lifshitz point. J. Chem. Phys. 110 (1999) 4079-4089.

[19] P.E. Kloeden and S. Shot, Linear-implicit strong schemes for Itô-Galerkin approximations of stochastic PDEs. J. Appl. Math. Stoch. Anal. 14 (2001) 47-53.

[20] G.T. Kossioris and G.E. Zouraris, Fully-Discrete Finite Element Approximations for a Fourth-Order Linear Stochastic Parabolic Equation with Additive Space-Time White Noise. TRITA-NA 2008:2, School of Computer Science and Communication, KTH, Stockholm, Sweden (2008).

[21] J.L. Lions and E. Magenes, Non-Homogeneous Boundary Value Problems and Applications, Vol. I. Springer-Verlag, BerlinHeidelberg, Germany (1972).

[22] T. Müller-Gronbach and K. Ritter, Lower bounds and non-uniform time discretization for approximation of stochastic heat equations. Found. Comput. Math. 7 (2007) 135-181.

[23] J. Printems, On the discretization in time of parabolic stochastic partial differential equations. ESAIM: M2AN 35 (2001) $1055-1078$

[24] V. Thomée, Galerkin Finite Element Methods for Parabolic Problems, Spriger Series in Computational Mathematics 25. Springer-Verlag, Berlin-Heidelberg, Germany (1997).

[25] Y. Yan, Error analysis and smothing properies of discretized deterministic and stochastic parabolic problems. Ph.D. Thesis, Department of Computational Mathematics, Chalmers University of Technology and Göteborg University, Göteborg, Sweden (2003).

[26] Y. Yan, Semidiscrete Galerkin approximation for a linear stochastic parabolic partial differential equation driven by an additive noise. BIT 44 (2004) 829-847.

[27] Y. Yan, Galerkin finite element methods for stochastic parabolic partial differential equations. SIAM J. Numer. Anal. 43 (2005) 1363-1384.

[28] J.B. Walsh, An introduction to stochastic partial differential equations., Lecture Notes in Mathematics 1180. Springer Verlag, Berlin-Heidelberg, Germany (1986) 265-439.

[29] J.B. Walsh, Finite element methods for parabolic stochastic PDEs. Potential Anal. 23 (2005) 1-43. 